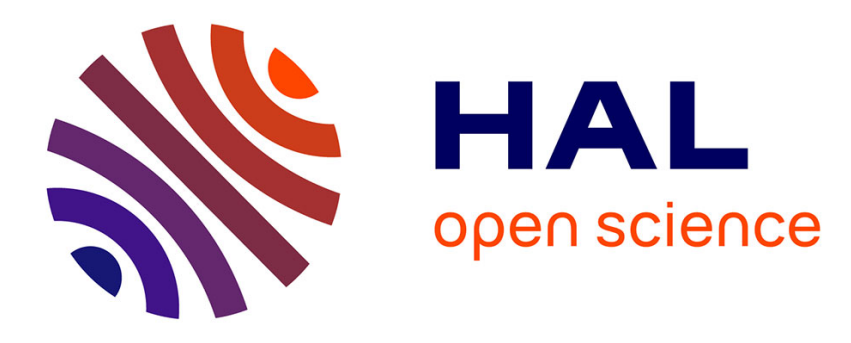

\title{
Polymers on disordered hierarchical lattices : a nonlinear combination of random variables
}

\author{
J. Cook, Bernard Derrida
}

\section{To cite this version:}

J. Cook, Bernard Derrida. Polymers on disordered hierarchical lattices : a nonlinear combination of random variables. Journal of Statistical Physics, 1989, 57 (1-2), pp.89-139. 10.1007/BF01023636 . hal-03285625

\section{HAL Id: hal-03285625 \\ https://hal.science/hal-03285625}

Submitted on 21 Jul 2021

HAL is a multi-disciplinary open access archive for the deposit and dissemination of scientific research documents, whether they are published or not. The documents may come from teaching and research institutions in France or abroad, or from public or private research centers.
L'archive ouverte pluridisciplinaire HAL, est destinée au dépôt et à la diffusion de documents scientifiques de niveau recherche, publiés ou non, émanant des établissements d'enseignement et de recherche français ou étrangers, des laboratoires publics ou privés. 


\title{
Polymers on Disordered Hierarchical Lattices: A Nonlinear Combination of Random Variables
}

\author{
J. Cook ${ }^{1,2}$ and B. Derrida ${ }^{1}$
}

Received February 24, 1989; revision received June 14, 1989

\begin{abstract}
The problem of directed polymers on disordered hierarchical and hypercubic lattices is considered. For the hierarchical lattices the problem can be reduced to the study of the stable laws for combining random variables in a nonlinear way. We present the results of numerical simulations of two hierarchical lattices, finding evidence of a phase transition in one case. For a limiting case we extend the perturbation theory developed by Derrida and Griffiths to nonzero temperature and to higher order and use this approach to calculate thermal and geometrical properties (overlaps) of the model. In this limit we obtain an interpolation formula, allowing one to obtain the noninteger moments of the partition function from the integer moments. We obtain bounds for the transition temperature for hierarchical and hypercubic lattices, and some similarities between the problem on the two different types of lattice are discussed.
\end{abstract}

KEY WORDS: Stable laws; disorder; polymer.

\section{INTRODUCTION}

The problem of a polymer in a random medium can be formulated in its simplest lattice version as follows. ${ }^{(1)}$ Consider a regular lattice with an energy $\varepsilon_{i j}$ chosen randomly according to a given probability distribution $\rho\left(\varepsilon_{i j}\right)$ on each bond $i j$ of the lattice. On this lattice, one would like to study the thermal properties of a self-avoiding walk (polymer) with a fixed origin $O$ and a given length $L$. By definition, the partition function $Z$ of this polymer at temperature $T$ is given by

$$
Z=\sum_{W} e^{-E_{W} / T}
$$

\footnotetext{
${ }^{1}$ Service de Physique Théorique de Saclay (Laboratoire de l'Institut de Recherche Fondamentale du Commissariat à l'Energie Atomique), F-91191 Gif sur Yvette, France.

${ }^{2}$ Department of Physics, University of Edinburgh, Edinburgh EH9 3JZ, United Kingdom.
} 
where in (1) the sum runs over all the possible self-avoiding walks of length $L$ starting at the origin $O$ and where the energy $E_{w}$ of each walk $W$ is the energy of all the bonds visited by the walk

$$
E_{W}=\sum_{i j} \varepsilon_{i j}
$$

As with other models in statistical mechanics, one would like to know the free energy of this system in the thermodynamic limit $(L \rightarrow \infty)$ in order to understand the phase diagram and the characteristics of each thermodynamic phase. In addition to the free energy, it is interesting to consider several other properties of this system (geometrical properties such as the average end-to-end distance of the walk, dynamical properties such as the diffusion constant, etc.).

This problem in the theory of disordered systems has been of interest for the last few years for several reasons:

1. It is a relevant question in the study of the properties of polymeric chains in porous media ${ }^{(2-12)}$ or of the pinning of one-dimensional interfaces by random impurities ${ }^{(12-19)}$ in two-dimensional systems. In its zero-temperature limit, the problem becomes an optimization problem: one has to find the best path $^{(20-23)}$ in a random environment.

2. The problem of a self-avoiding walk on a random lattice can be viewed as the limiting case of a magnetic system with random interactions $^{(4,5)}$ (in the limit of spins with zero component). The corresponding pure system (all the $\varepsilon_{i j}$ equal) has a positive specific heat exponent $\alpha$ and one expects that any amount of disorder would change the critical properties of the walk.

3. There is a strong analogy between the problem of a polymer in a random medium and spin glasses. ${ }^{(1)}$ One might then wonder whether some properties specific to spin glasses (multivalley landscape, broken replica symmetry, non-self-averaging effects) could also be observed in the case of polymers in random media.

Although the above questions have not yet been fully answered, some progress has been made in the last few years.

First, by considering a simplified version of the problem, that of directed polymers (i.e., walks such that one of their coordinates is an increasing function of time), ${ }^{(21,23,24)}$ it is possible to obtain exact results ${ }^{(13,21,2426)}$ in dimension $2=1+1$ (for directed polymers, the direction along which the polymer is directed always plays a special role).

Second, in $d>3(=2+1)$, it is possible to prove that at high enough temperature, ${ }^{(27,28)}$ the quenched free energy $\langle\log Z\rangle$ of the directed walk is 
identical to the annealed free energy $\log \langle Z\rangle$ (which can be calculated easily; see Section 9). This implies that there is a high-temperature phase where the problem is well understood.

Third, in the mean-field limit $(d \rightarrow \infty)$, the problem can be solved exactly (there is a very simple expression of the free energy). ${ }^{(1)}$ One finds that there exists a transition temperature below which the system has properties very similar to those predicted for mean-field spin glasses ${ }^{(29-31)}$ (broken replica symmetry, non-self-averaging effects, a nontrivial probability distribution for the overlaps, etc.).

Due to this analogy, it is legitimate to wonder whether all the properties characteristic of the mean-field spin glass are present only for the polymer problem in infinite dimension or whether they persist in finite dimension.

The study of directed polymers with disorder in arbitrary dimension is still a very open question and the nature of the low-temperature phase is not yet well understood. Recently a related problem has been investigated which consists in considering polymers on a hierarchical lattice in the presence of disorder. ${ }^{(23)}$ This is a much simpler problem than the finitedimensional one because it is possible to write simple recursions for the partition function. It is, however, reasonable to think that hierarchical lattices are much closer to finite-dimensional systems than mean-field models, in particular because one can vary some parameters which define the hierarchical lattice in order to go from low- to high-dimensional systems.

The main purpose of the present work is to extend to finite temperature some analytical results which have been obtained recently for the problem of polymers on disordered hierarchical lattices. ${ }^{(23)}$ We will show that quantities such as the free energy, overlaps of an arbitrary number of configurations, the probability distribution of overlaps, ${ }^{(29,31)}$ and integer and noninteger moments of the partition function can be calculated at any temperature by a perturbation method.

As we will see in Section 2, the problem of polymers on a random hierarchical lattice is closely related to an interesting problem in probability theory: the problem of finding stable laws when one combines random variables in a nonlinear way; for example, if one combines $a b$ random variables $Z_{n}^{(i)}$ to obtain a new variable $Z_{n+1}$ given by

$$
Z_{n+1}=Z_{n}^{(1)} Z_{n}^{(2)} \cdots Z_{n}^{(a)}+Z_{n}^{(a+1)} \cdots Z_{n}^{(2 a)}+\cdots+Z_{n}^{(a b-a+1)} \cdots Z_{n}^{(a b)}
$$

( $Z_{n+1}$ is the sum of $b$ products of $a$ random variables), the problem is to find a stable law for this operation, i.e., the shape of a distribution which remains unchanged when one goes from the $Z_{n}^{(i)}$ to $Z_{n+1}$. This problem has 
a simple answer if $a=1$ or $b=1$ (for $a=1$ the stable law is a Gaussian distribution, whereas for $b=1$ it is a $\log$ normal distribution), but is much more difficult if both $a$ and $b$ are larger than 1 .

This paper is organized as follows. In Section 2 we define the model of polymers on a random hierarchical lattice and we show that it can be reduced to the study of recursions similar to (3). In Section 3, we calculate a few integer moments and we obtain the phase diagram and the overlaps corresponding to these moments. In Section 4, we describe a Monte Carlo method which gives numerical estimates of the thermal properties and of the overlaps. In Section 5, we present a perturbative approach which allows one to calculate analytically the free energy at any temperature. In the zero-temperature limit, we recover the results of ref. 23. In Sections 6 and 7 , we calculate the overlaps and the (integer and noninteger) moments of $Z$ and we discuss the validity of the replica method (we will see that the zero-temperature limit and the limit of zero replica do not commute). In Section 8 we show how the results obtained in Sections 5-7 can be extended to other hierarchical structures. Lastly, in Section 9, we compare some properties of the problem on a hierarchical lattice and on a hypercubic lattice.

\section{CONSTRUCTION OF THE HIERARCHICAL LATTICE AND OF THE ITERATIVE RULES}

As in ref. 23, we will consider here a family of hierarchical lattices which are constructed by an iterative rule. The rule consists of building the lattice at the $(n+1)$ th generation by replacing each bond of the lattice at the $n$th generation by a motif of $2 b$ bonds as shown in Fig. 1. The so-called diamond lattice (which is the case $b=2$ ) is shown in Fig. 1a, and the generalization of it (arbitrary $b$ ) is shown in Fig. $1 b$.

Since at each new generation the number of bonds is multiplied by $2 b$, the hierarchical lattice at the $n$th generation consists of $(2 b)^{n-1}$ bonds. As for the other lattice models, one assigns a random energy $\varepsilon_{i j}$ chosen according to a given probability distribution $\rho\left(\varepsilon_{i j}\right)$ to each bond $i j$ of the lattice. Although several results which will be discussed in what follows could be obtained for arbitrary distributions $\rho(\varepsilon)$, we will mostly limit our discussion to a Gaussian distribution

$$
\rho(\varepsilon)=(2 \pi)^{-1 / 2} \exp \left(-\varepsilon^{2} / 2\right)
$$

On this hierarchical lattice (Fig. 1), we consider all the directed walks starting from point $A$ and ending at point $B$. On a lattice at generation $n$, there are $b^{2^{n-1}-1}$ such walks and the length of each of these walks is $L=2^{n-1}$. 


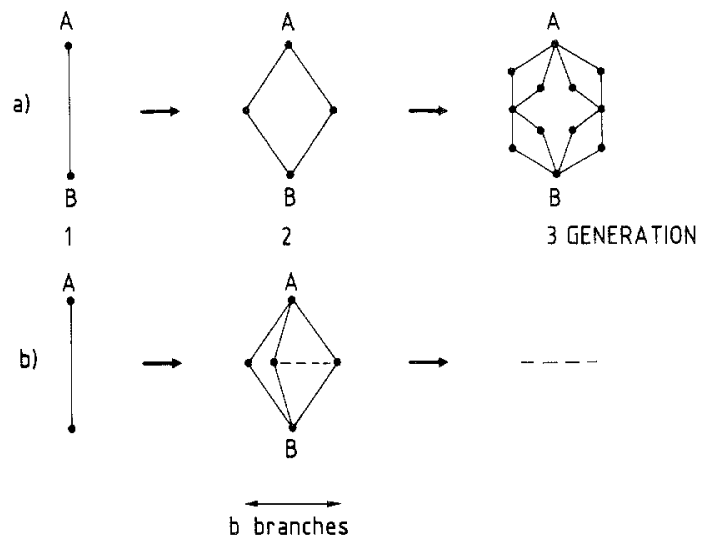

Fig. 1. The iterative construction of (a) the diamond hierarchical lattice and (b) the generalized lattice.

Because the lattice at generation $n+1$ can always be viewed as the combination of $2 b$ lattices at generation $n$, one can write the following recursion for the partition function:

$$
Z_{n+1}=Z_{n}^{(1)} Z_{n}^{(2)}+\cdots+Z_{n}^{(2 b-1)} Z_{n}^{(2 b)}
$$

where $Z_{n+1}$ is the partition function of a lattice at the $(n+1)$ th generation and the $Z_{n}^{(i)}$ are the partition functions of $2 b$ (different) lattices at generation $n$.

Because all the partition functions which appear in (5) are random, the quantity one needs to consider is the probability distribution $\pi_{n}(Z)$ of the partition function $Z$ of a lattice at the $n$th generation. Clearly (5) implies that

$$
\pi_{n+1}(Z)=\int \cdots \int \pi_{n}\left(Z_{1}\right) d Z_{1} \cdots \pi_{n}\left(Z_{2 b}\right) d Z_{2 b} \delta\left(Z-\left[Z_{1} Z_{2}+\cdots+Z_{2 b-1} Z_{2 b}\right]\right)
$$

Since at the first generation, the lattice consists of a single bond, one can write that

$$
\pi_{1}(Z)=\int \rho(\varepsilon) d \varepsilon \delta\left(Z-e^{-\varepsilon / T}\right)=\frac{T}{Z} \rho(-T \log Z)
$$

So the problem of calculating the free energy is reduced to the study of the recursion (6) for the probability distribution $\pi_{n}(Z)$, and the average free 
energy $F_{n}(T)$ per unit length of the walk on a lattice at the $n$th generation will be given by

$$
F_{n}(T)=-T \frac{\left\langle\log Z_{n}\right\rangle}{2^{n-1}}=-\frac{T}{2^{n-1}} \int \pi_{n}(Z) d Z \log Z
$$

where $\langle\cdot\rangle$ denotes the average over disorder.

Apart from the thermal properties of the walk (energy, specific heat), which can be computed as derivatives of $F_{n}(T)$ with respect to temperature, it is interesting to study other properties of the walk, such as the overlaps.

If one considers two walks $W$ and $W^{\prime}$ of length $L=2^{n-1}$ on the same random lattice (at generation $n$ ), one can define the overlap $q_{2}\left(W, W^{\prime}\right)$ of these two walks as the fraction of their length they spend together. This definition is the direct generalization to this problem of the notion of overlaps which is used in spin glasses. ${ }^{(30,31)}$ From this definition of overlaps, one can introduce the probability $P_{2}(q)$ of finding two configurations with an overlap $q$ :

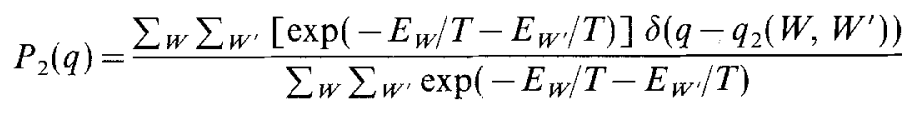

As in Eq. (5), it is possible to obtain $P_{2}(q)$ from a recursion relation. To do this, it is convenient to introduce the following generating function $X_{n}(T, y)$ for a lattice at the $n$th generation:

$$
X_{H}(T, y)=\sum_{W} \sum_{W^{\prime}} \exp \left[-\frac{E_{W}}{T}-\frac{E_{W^{\prime}}}{T}+y 2^{n-1} q_{2}\left(W, W^{\prime}\right)\right]
$$

Then one can write the following recursion formula for $X$ :

$$
\begin{aligned}
X_{n+1}= & X_{n}^{(1)} X_{n}^{(2)}+\cdots+X_{n}^{(2 b-1)} X_{n}^{(2 b)} \\
& +\left[Z_{n}^{(1)} Z_{n}^{(2)}+\cdots+Z_{n}^{(2 b-1)} Z_{n}^{(2 b)}\right]^{2} \\
& -\left[\left(Z_{n}^{(1)} Z_{n}^{(2)}\right)^{2}+\cdots+\left(Z_{n}^{(2 b-1)} Z_{n}^{(2 b)}\right)^{2}\right]
\end{aligned}
$$

The origin of this relation is very similar to the origin of Eq. (5): the lattice at the $(n+1)$ th generation is composed of $2 b$ lattices at the $n$th generation. If one considers two walks on the lattice at the $(n+1)$ th generation, either they go through the same branch [first term of the rhs of (11)] or they go through different branches [last two terms of (11)]. Then in principle one should consider the probability $\tilde{\pi}_{n}(X, Z)$ of $X$ and $Z$ and write an iterative formula similar to $(6)$ with the following initial condition:

$$
\tilde{\pi}_{1}(X, Z)=\int \rho(\varepsilon) d \varepsilon \delta\left(X-e^{y-2 \varepsilon / T}\right) \delta\left(Z-e^{-\varepsilon / T}\right)
$$


which expresses the fact that at the first generation, the lattice consists of a single bond and that two walks on this lattice are identical and have the same energy. If one can calculate $\tilde{\pi}_{n}(X, Z)$, then the average $\left\langle X_{n}(T, y) / Z_{n}^{2}(T)\right\rangle$ over disorder is known and one can deduce the average overlap $q_{2}$ by

$$
q_{2}(T)=\left.\frac{1}{2^{n-1}} \frac{d}{d y}\left\langle\frac{X_{n}(T, y)}{Z_{n}^{2}(T)}\right\rangle\right|_{y=0}
$$

The procedure that we have just described to calculate $q_{2}(T)$ can easily be extended to obtain other overlaps, such as the overlap $q_{m}\left(W^{(1)}, \ldots, W^{(m)}\right)$ between $m$ walks (this overlap is simply the fraction of their length that these $m$ walks spend together). Defining, as in (10), $X_{n}(T, y)$ by

$$
X_{n}(T, y)=\sum_{W^{(1)}} \sum_{W^{(2)}} \cdots \sum_{W^{(m)}} \exp \left[-\frac{Z_{W^{(1)}}}{T} \cdots-\frac{E_{W^{(m)}}}{T}+y 2^{n-1} q_{m}\left(W^{(1)}, \ldots, W^{(m)}\right)\right]
$$

One can write a recursion very similar to (11)

$$
\begin{aligned}
X_{n+1}= & X_{n}^{(1)} X_{n}^{(2)}+\cdots+X_{n}^{(2 b-1)} X_{n}^{(2 b)} \\
& +\left(Z_{n}^{(1)} Z_{n}^{(2)}+\cdots Z_{n}^{(2 b-1)} Z_{n}^{(2 b)}\right)^{m} \\
& -\left[\left(Z_{n}^{(1)} Z_{n}^{(2)}\right)^{m}+\cdots+\left(Z_{n}^{(2 b-1)} Z_{n}^{(2 b)}\right)^{m}\right]
\end{aligned}
$$

with the initial condition

$$
X_{1}^{(i)}=e^{y}\left(Z_{1}^{(i)}\right)^{m}
$$

We see from (5), (11), and (15) that the problem of a polymer on a random hierarchical lattice can be understood if one knows how to describe the probability distribution of variables like $X_{n+1}$ or $Z_{n+1}$ which are obtained by combining other random variables (the $X_{n}^{(i)}$ and the $Z_{n}^{(i)}$ ) in a nonlinear way. Of course, if $b=1, \log Z_{n}$ is a sum of random variables and the solution is given by the central limit theorem. However, as soon as $b>1$, the problem is much more complex and, to our knowledge, the shape of the limiting distribution of $Z_{n}$ when $n \rightarrow \infty$ is unknown.

The problem of finding the distribution of $Z_{n}$ in the limit $n \rightarrow \infty$ is very similar to the distribution of ground-state energies of the same problem. ${ }^{(23)}$ At zero temperature, the recursion relation (5) becomes a recursion relation for the ground-state energy $E_{n}$ of a lattice at the $n$th generation

$$
E_{n+1}=\min \left[E_{n}^{(1)}+E_{n}^{(2)}, \ldots, E_{n}^{(2 b-1)}+E_{n}^{(2 b)}\right]
$$


Then one can write the following recursion for the probablity distribution $P_{n}\left(E_{n}\right)$ of $E_{n}$ :

$$
\begin{gathered}
P_{n+1}(E)=b Q_{n}(E)\left[\int_{E}^{\infty} d E^{\prime} Q_{n}\left(E^{\prime}\right)\right]^{b-1} \\
Q_{n}(E)=\int d E^{\prime} P_{n}\left(E^{\prime}\right) P_{n}\left(E-E^{\prime}\right)
\end{gathered}
$$

In ref. 23, numerical and analytical results were presented concerning the iteration (18). For large $n$, it was observed numerically than $P_{n}(E)$ takes the following form:

$$
P_{n}(E)=\frac{1}{\Delta_{n}} F_{b}\left(\frac{E-\gamma_{n}}{\Delta_{n}}\right)
$$

where $\gamma_{n}$ and $\Delta_{n}$ both depend on the initial distribution $P_{1}(E)=\rho(E)$, but $F_{b}$ does not. Moreover, for $n \rightarrow \infty$, the ratio $\Delta_{n+1} / \Delta_{n}$ has a limit $\lambda_{b}$ independent of the initial distribution $P_{1}(E)$. This implies that the fluctuations of the ground-state energy increase with the length $L$ of the polymer as a power law

$$
\left[\left\langle E_{n}^{2}\right\rangle-\left\langle E_{n}\right\rangle^{2}\right]^{1 / 2} \sim \Delta_{n} \sim\left(\lambda_{b}\right)^{n} \sim L^{\omega}
$$

with

$$
\omega=\log \lambda_{b} / \log 2
$$

The ground-state energy per unit length of the polymer $\left\langle E_{n}\right\rangle / 2^{n-1}$ and the scaling factor $\lambda_{b}$ were measured numerically for several values of $b$ and it was shown that one can develop a perturbative approach for $b$ close to 1 which gives analytic expressions for these two quantities.

\section{THE INTEGER MOMENTS}

For most disordered systems, the calculation of integer moments of the partition function is much easier than that of the average free energy.

For the problem of directed polymers, it is easy to see that the calculation of the integer moments can be reduced to the study of simple iterations. From (5), one can obtain the following recursion relations for the first moments of $Z_{n}$ :

$$
\begin{aligned}
& \left\langle Z_{n+1}\right\rangle=b\left\langle Z_{n}\right\rangle^{2} \\
& \left\langle Z_{n+1}^{2}\right\rangle=b\left\langle Z_{n}^{2}\right\rangle^{2}+b(b-1)\left\langle Z_{n}\right\rangle^{4}
\end{aligned}
$$




$$
\begin{aligned}
\left\langle Z_{n+1}^{3}\right\rangle= & b\left\langle Z_{n}^{3}\right\rangle^{2}+3 b(b-1)\left\langle Z_{n}^{2}\right\rangle^{2}\left\langle Z_{n}\right\rangle^{2}+b(b-1)(b-2)\left\langle Z_{n}\right\rangle^{6} \\
\left\langle Z_{n+1}^{4}\right\rangle= & b\left\langle Z_{n}^{4}\right\rangle^{2}+4 b(b-1)\left\langle Z_{n}^{3}\right\rangle^{2}\left\langle Z_{n}\right\rangle^{2}+3 b(b-1)\left\langle Z_{n}^{2}\right\rangle^{4} \\
& +6 b(b-1)(b-2)\left\langle Z_{n}^{2}\right\rangle^{2}\left\langle Z_{n}\right\rangle^{4}+b(b-1)(b-2)(b-3)\left\langle Z_{n}\right\rangle^{8}
\end{aligned}
$$

with the initial condition

$$
\left\langle Z_{1}^{p}\right\rangle=\mu_{p}=\int \rho(\varepsilon) e^{-p \varepsilon / T} d \varepsilon
$$

To calculate the $p$ th moment of $Z_{n}$, one needs to study a simple dynamical system in a $p$-dimensional space, since the calculation of the $p$ th moment at the $(n+1)$ th generation only requires the knowledge of the first $p$ moments at the $n$th generation. This dynamical system can be simplified further by considering the ratios

$$
z_{p}(n)=\left\langle Z_{n}\right\rangle^{p} /\left\langle Z_{n}^{p}\right\rangle
$$

and the recursions (23)-(26) become

$$
\begin{aligned}
z_{2}(n+1)= & b\left[z_{2}(n)\right]^{2}\left\{1+(b-1)\left[z_{2}(n)\right]^{2}\right\}^{-1} \\
z_{3}(n+1)= & b^{2}\left[z_{3}(n)\right]^{2}\left\{1+3(b-1)\left(\frac{z_{3}(n)}{z_{2}(n)}\right)^{2}+(b-1)(b-2)\left[z_{3}(n)\right]^{2}\right\}_{(29}^{-1} \\
z_{4}(n+1)= & b^{3}\left[z_{4}(n)\right]^{2}\left\{1+4(b-1)\left(\frac{z_{4}(n)}{z_{3}(n)}\right)^{2}+3(b-1) \frac{\left[z_{4}(n)\right]^{2}}{\left[z_{2}(n)\right]^{4}}\right. \\
& \left.+6(b-1)(b-2)\left(\frac{z_{4}(n)}{z_{2}(n)}\right)^{2}+(b-1)(b-2)(b-3)\left[z_{4}(n)\right]^{2}\right\}^{-1}
\end{aligned}
$$

There exist several attractive fixed points of these recursions and each possible phase of $\left\langle Z^{p}\right\rangle$ corresponds to one of these attractive fixed points.

To describe the phase diagram of $\left\langle Z^{2}\right\rangle$, it is sufficient to study the recursion (28). There are three fixed points of (28): $z_{2}=1, z_{2}=0$, and $z_{2}=$ $1 /(b-1)$. For $b \leqslant 2, z_{2}=0$ is attractive and $z_{2}=1$ is unstable, whereas $z_{2}=$ $1 /(b-1)$ is unreachable if $0<z_{2}(1)<1$ [see (27)]. So any initial $z_{2}(1) \neq 1$ converges to $z_{2}=0$. For $b>2$ the two fixed points $z_{2}=1$ and $z_{2}=0$ are attractive and the fixed point $z_{2}=1 /(b-1)$ is unstable. Depending on the initial value $z_{2}(1)$, the point $z_{2}(n)$ converges to one of these two attractive 
fixed points: $z_{2}(n) \rightarrow 0$ if $z_{2}(1)<1 /(b-1)$ and $z_{2}(n) \rightarrow 1$ if $z_{2}(1)>1 /(b-1)$. So we see that in the plane $(b, T)$, the line $T_{2}(b)$ given by

$$
z_{2}(1)=\frac{\left[\int \rho(\varepsilon) \exp \left(-\varepsilon / T_{2}\right) d \varepsilon\right]^{2}}{\int \rho(\varepsilon) \exp \left(-2 \varepsilon / T_{2}\right) d \varepsilon}=\frac{1}{b-1}
$$

is a transition line for $\left\langle Z^{2}\right\rangle$. For the Gaussian distribution (4), this line $T_{2}(b)$ is given by

$$
T_{2}(b)=[\log (b-1)]^{-1 / 2}
$$

and is shown on Fig. 2. We see that $T_{2}(b)$ diverges as $b \rightarrow 2$ and that for $b<2,\left\langle Z^{2}\right\rangle$ is always in its low-temperature phase. For $T>T_{2}(b)$, $z_{2}(n) \rightarrow 1$ as $n \rightarrow \infty$, and so one knows that $\left\langle Z_{n}^{2}\right\rangle /\left\langle Z_{n}\right\rangle^{2} \rightarrow 1$ and this implies that the distribution of $Z_{n}$ becomes very narrow arround $\left\langle Z_{n}\right\rangle$. As a consequence, for $T>T_{2}(b)$, one has

$$
\lim _{n \rightarrow \infty} \frac{1}{2^{n-1}} \log Z_{n}=\lim _{n \rightarrow \infty} \frac{1}{2^{n-1}} \log \left\langle Z_{n}\right\rangle
$$

with probability 1 .

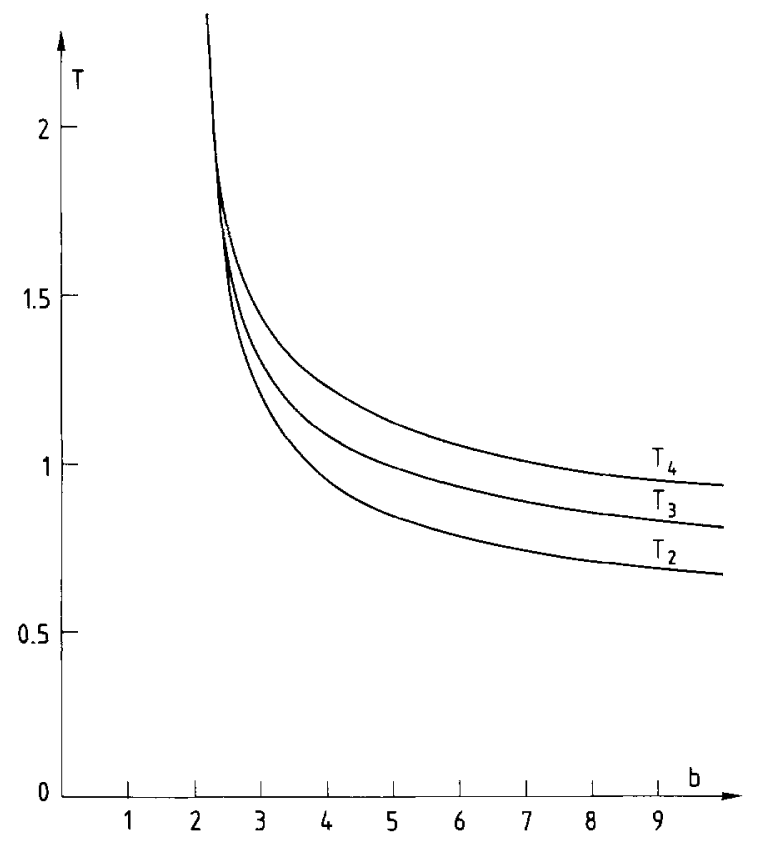

Fig. 2. The transition temperatures $T_{2}(b), T_{3}(b)$, and $T_{4}(b)$ of the moments $\left\langle Z^{2}\right\rangle,\left\langle Z^{3}\right\rangle$, and $\left\langle Z^{4}\right\rangle$ versus $b$. 
From (22) one can easily calculate $\left\langle Z_{n}\right\rangle$ for the Gaussian distribution (4),

$$
\left\langle Z_{n}\right\rangle=b^{2^{n-1}-1} e^{2^{n-2} / T^{2}}
$$

and one can deduce from (33) that for $T>T_{2}(b)$ the free energy $F_{n}(T)$ per unit length is given with probability one by

$$
\lim _{n \rightarrow \infty} F_{n}(T)=\lim _{n \rightarrow \infty}-T \frac{\log Z_{n}}{2^{n-1}}=-T\left(\log b+\frac{1}{2 T^{2}}\right)
$$

It is interesting to recover here a feature which has been found in the study of directed polymers in finite dimensions ${ }^{(27,28)}$ : above a certain temperature $T_{2}(b)$, the free energy is known exactly (see Section 9). One should notice that the fact that $\left\langle Z_{n}^{2}\right\rangle /\left\langle Z_{n}\right\rangle^{2} \rightarrow 1$ as $n \rightarrow \infty$ implies that $\log Z_{n}$ is equal to $\log \left\langle Z_{n}\right\rangle$ with probability one. However, it could happen that $\left\langle\log Z_{n}\right\rangle \neq \log \left\langle Z_{n}\right\rangle$ due to rare events with $Z_{n}$ close to 0.

One should notice that the fact that we know that (35) is valid above $T_{2}(b)$ does not imply that the transition temperature $T_{c}(b)$ is $T_{2}(b)$. This is because (33) might remain true even when $\left\langle Z_{n}\right\rangle^{2} /\left\langle Z_{n}^{2}\right\rangle \rightarrow 0$. However, it is easy to see that (35) cannot remain true down to $T=0$ [the entropy calculated from (35) would vanish ${ }^{(32,33)}$ at $\left.T=(2 \log b)^{-1 / 2}\right]$. This implies that $\log Z_{n}$ must undergo a transition above this temperature. Therefore, the free energy is given with probability one by (35) down to a certain transition temperature $T_{c}(b)$ for which we have the following bounds:

$$
(2 \log b)^{-1 / 2} \leqslant T_{c}(b) \leqslant[\log (b-1)]^{-1 / 2}
$$

By looking at the two-dimensional mapping $\left(z_{2}(n), z_{3}(n)\right) \rightarrow$ $\left(z_{2}(n+1), z_{3}(n+1)\right)$, Eq. (29), one can get the phase diagram of $\left\langle Z_{n}^{3}\right\rangle$. The analytic form of the transition line $T_{3}(b)$ is not as simple as (32): one can show (but it would be too long to give the explanation here) that for $b<2.303 \ldots, T_{3}(b)=T_{2}(b)$, whereas for $b>2.303, T_{3}(b)$ is obtained by the condition that the point $\left(z_{2}(1), z_{3}(1)\right)$ lies on the stable manifold of the fixed point $\left(z_{2}=1, z_{3}=1 /\left(b^{2}-1\right)\right)$. The transition line $T_{3}(b)$ versus $b$ is shown on Fig. 2.

In a similar way, the transition temperature of $\left\langle Z^{4}\right\rangle$ can be obtained by studying the three-dimensional mapping $\left(z_{2}(n), z_{3}(n), z_{4}(n)\right) \rightarrow$ $\left(z_{2}(n+1), z_{3}(n+1), z_{4}(n+1)\right)$. One finds a transition temperature $T_{4}(b)$ shown on Fig. 2. As for $T_{3}(b), T_{4}(b)$ coincides with $T_{2}(b)$ for $b$ close enough to 2 and then it is given by the condition that the point $\left(z_{2}(1)\right.$, $\left.z_{3}(1), z_{4}(1)\right)$ lies on the stable manifold of the fixed point $\left(z_{2}=1, z_{3}=1\right.$, $\left.z_{4}=1 /\left(b^{3}-1\right)\right)$. 
Looking at Fig. 2, one expects that higher integer moments would have higher transition temperatures $T_{n}(b)$.

The main reason that it is interesting to calculate the moments $\left\langle Z_{n}^{p}\right\rangle$ is to try to use a replica approach. In principle, if one could calculate $\psi_{p}(n)$ defined by

$$
\psi_{p}(n)=\frac{1}{2^{n-1}} \log \left\langle\left(Z_{n}\right)^{p}\right\rangle
$$

for all $p$, the replica approach would give the average free energy $F_{n}(T)$ by

$$
F_{n}(T)=\frac{-T}{2^{n-1}}\left\langle\log Z_{n}\right\rangle=-T \lim _{p \rightarrow 0} \frac{\psi_{p}(n)}{p}
$$

From the previous relations (22)-(30), one can get expressions for the $\psi_{p}(n)$ for $p=1,2,3$, and 4 . One finds, as usual for models on hierarchical lattices, ${ }^{(34,35)}$ that the results are given by a sum

$$
\begin{aligned}
\psi_{1}(n)= & \left(1-\frac{1}{2^{n-1}}\right) \log b+\frac{1}{2 T^{2}} \\
\psi_{2}(n)= & \left(1-\frac{1}{2^{n-1}}\right) \log b+\frac{4}{2 T^{2}} \\
& +\sum_{i=1}^{n-1} \frac{1}{2^{i-1}} \log \left\{1+(b-1)\left[z_{2}(i)\right]^{2}\right\} \\
\psi_{3}(n)= & \left(1-\frac{1}{2^{n-1}}\right) \log b+\frac{9}{2 T^{2}} \\
& +\sum_{i=1}^{n-1} \frac{1}{2^{i-1}} \log \left\{1+3(b-1)\left(\frac{z_{3}(i)}{z_{2}(i)}\right)^{2}+(b-1)(b-2)\left[z_{3}(i)\right]^{2}\right\} \\
\psi_{4}(n)= & \left(1-\frac{1}{2^{n-1}}\right) \log b+\frac{16}{2 T^{2}} \\
& +\sum_{i=1}^{n-1} \frac{1}{2^{i-1}} \log \left\{1+4(b-1)\left(\frac{z_{4}(i)}{z_{3}(i)}\right)^{2}\right. \\
& +3(b-1) \frac{\left[z_{4}(i)\right]^{2}}{\left[z_{2}(i)\right]^{4}}+6(b-1)(b-2)\left(\frac{z_{4}(i)}{z_{2}(i)}\right)^{2} \\
& \left.+(b-1)(b-2)(b-3)\left[z_{4}(i)\right]^{2}\right\}
\end{aligned}
$$


Looking at these expressions, it is easy to guess an extrapolation to arbitrary $p$ of the first two terms of the right-hand sides of (39)-(42). However, for the last term, this extrapolation seems much more difficult. We will see in Sections 5 and 6 that at least for $b$ close to 1 , one can calculate the moments for arbitrary (integer and noninteger) $p$ and thus that this extrapolation can be done when one expands around $b=1$.

Exactly as it was possible to deduce the recursion (22)-(25) from (5), one can also write when $p$ is an integer $\geqslant 2$ a recursion for $\phi_{n}(p)$ which is defined by

$$
\phi_{n}(p)=\left\langle X_{n}\left(Z_{n}\right)^{p-2}\right\rangle
$$

where $X_{n}$ is defined by (10). For arbitrary $p$, the knowledge of $\phi_{n}(p)$ would give the overlap $Q_{n}(p)$ between two walks among $p$ walks by the formula

$$
Q_{n}(p)=\left.\frac{d}{d y} \log \left[\phi_{n}(p)\right]\right|_{y=0}
$$

and the overlap $q_{2}(T)$ of Eq. (13) could be calculated by

$$
q_{2}(T)=\lim _{p \rightarrow 0} Q_{n}(p)
$$

For $p=2,3,4$ one finds that the overlap between two configurations satisfies

$$
\begin{aligned}
Q_{n}(2)= & \prod_{i=1}^{n-1} \frac{1}{1+(b-1)\left(z_{2}\right)^{2}} \\
Q_{n}(3)= & \prod_{i=1}^{n-1} \frac{1+(b-1)\left(z_{3} / z_{2}\right)^{2}}{1+3(b-1)\left(z_{3} / z_{2}\right)^{2}+(b-1)(b-2)\left(z_{3}\right)^{2}} \\
Q_{n}(4)= & \prod_{i=1}^{n-1}\left[1+2(b-1)\left(\frac{z_{4}}{z_{3}}\right)^{2}+(b-1) \frac{\left(z_{4}\right)^{2}}{\left(z_{2}\right)^{4}}+(b-1)(b-2)\left(\frac{z_{4}}{z_{2}}\right)^{2}\right] \\
& \times\left[1+4(b-1)\left(\frac{z_{4}}{z_{3}}\right)^{2}+3(b-1) \frac{\left(z_{4}\right)^{2}}{\left(z_{2}\right)^{4}}+6(b-1)(b-2)\left(\frac{z_{4}}{z_{2}}\right)^{2}\right. \\
& \left.+(b-1)(b-2)(b-3)\left(z_{4}\right)^{2}\right]^{-1}
\end{aligned}
$$

where in (46)-(48) $z_{2}=z_{2}(i), z_{3}=z_{3}(i)$, and $z_{4}=z_{4}(i)$. As for the expressions (39)-(42), it is not obvious from these expressions what the generalized expression for arbitrary $p$ would be.

It is clear that expressions similar to (46)-(48) could be found for overlaps of more than two walks. Again looking at the expressions for the 
overlaps of $m$ walks for $p=3,4$, it is hard to guess an expression valid for arbitrary $p$.

We have seen in this section that several properties concerning the integer moments of $Z$ can be calculated on the hierarchical lattice. The calculation of the second moment allowed us to obtain the exact expression (35) for the free energy above the temperature $T_{2}(b)$ given by (32). The exact expressions for the first moments (39)-(42) or for the overlaps associated with these first moments (46)-(48) were, however, too complicated to allow us to guess a generalization of these expressions to noninteger moments. We will see in Sections 5-7 that such generalizations can be obtained at least in a perturbative way for $b$ close to 1 .

\section{MONTE CARLO SIMULATION OF THE SPECIFIC HEAT AND OF THE OVERLAPS}

Despite the simplicity of the recursion (5), we do not know any analytic way of calculating $\pi_{n}(Z)$ [defined by (6)] for arbitrary $n$ and $b$ to obtain the free energy $\left\langle\log Z_{n}\right\rangle$ as a function of temperature. As we will discuss in the next sections, we were only able to get an analytic expression in the limit $b$ close to 1 .

For arbitrary $b$, it is, however, possible to estimate the quantities of interest rather accurately by numerical methods. The method we use here is a Monte Carlo sampling which has the advantage of being easy to program. The idea is the following: although we cannot calculate $\pi_{n}(Z)$ analytically, we can sample it. So we can represent $\pi_{n}(Z)$ by $N\left(N=5 \times 10^{4}\right.$ or $2 \times 10^{5}$ ) different values of $Z: Z_{n}^{(1)}, \ldots, Z_{n}^{(N)}$. For example, to represent $\pi_{1}(Z)$, we choose $N$ random Gaussian numbers $\varepsilon_{i}$ and we obtain $N$ values $Z_{1}^{(i)}$ by

$$
Z_{1}^{(i)}=\exp \left(-\varepsilon_{i} / T\right)
$$

Then, to represent $\pi_{2}(Z)$ by $N$ values $Z_{2}^{(i)}$, we choose for each $i, 2 b$ random indices $j_{1}(i), \ldots, j_{2 b}(i)$ between 1 and $N$ and we calculate $Z_{2}^{(i)}$ by

$$
Z_{n+1}^{(i)}=Z_{n}^{\left(j_{1}\right)} Z_{n}^{\left(j_{2}\right)}+\cdots+Z_{n}^{\left(j_{2 b-1}\right)} Z_{n}^{(j 2 b)}
$$

for $n=1$. Once the $N$ values $Z_{2}^{(i)}$ have been calculated this way, one can calculate $N$ values $Z_{3}^{(i)}$ to represent $\pi_{3}(Z)$. Again one chooses for each $i, 2 b$ new random indices $j_{1}(i), \ldots, j_{2 b}(i)$ and one calculates each $Z_{3}^{(i)}$ by (50). One can iterate this procedure many times and at the $n$th step, one gets $N$ values $Z_{n}^{(i)}(1 \leqslant i \leqslant N)$ which represent the distribution $\pi_{n}(Z)$. 
Then if one needs to estimate the average of a function of $Z$, averaged over $\pi_{n}(Z)($ e.g., $\log Z)$, one replaces it by the average over the $Z_{n}^{(i)}$ :

$$
\int \log Z \pi_{n}(Z) d Z \simeq \frac{1}{N} \sum_{i=1}^{N} \log \left(Z_{n}^{(i)}\right)
$$

In practice, we often need the first or the second derivative of $Z$ with respect to temperature, $Z^{\prime}$ and $Z^{\prime \prime}$ (to obtain the energy or the specific heat). It is easy to write from (50) the recursion for these derivatives and at each step one represents the distribution by $N$ triplets $\left(Z_{n}^{(i)}, d Z_{n}^{(i)} / d T\right.$, $d^{2} Z_{n}^{(i)} / d T^{2}$ ) which are calculated by the same procedure.

The curves of the specific heat obtained for a lattice built up to the $n=16$ generation for $b=2$ and $b=5$ (for $N=5 \times 10^{4}$ and $2 \times 10^{5}$ ) are shown in Figs. $3 a$ and $3 b$. We see that for $b=2$, the specific heat is a smooth function of temperature, whereas for $b=5$ there is a sharp maximum at a temperature $T_{c}=0.75 \pm 0.05$ which seems to be strictly smaller than $T_{2} \simeq 0.8493$.

Above $T_{c}$, the numerical data agree well with the prediction

$$
C=1 / T^{2}
$$

for the specific heat per unit length which follows from (35). For $b=2$ the specific heat can be seen to approach the form given by (52). It is not clear whether the specific heat has the form (52) above a certain finite transition temperature $T_{c}$ or whether $T_{c}$ is infinite. The same procedure can be used to obtain the overlaps. As discussed in Section 2, one has to calculate the $Z_{n}$ and $X_{n}$ through (5) and (10) in order to obtain $\pi_{n}(X, Z)$. Our procedure consists in representing $\pi_{n}(X, Z)$ by $N$ pairs $\left(X_{n}^{(i)}\right.$ and $\left.Z_{n}^{(i)}\right)$. Thus, we calculate each pair $\left(X_{n+1}^{(i)}\right.$ and $\left.Z_{n+1}^{(i)}\right)$ by choosing for each $i, 2 b$ ancestors $j_{1}, \ldots, j_{2 b}$ and by using $(50)$ and

$$
\begin{aligned}
X_{n+1}^{(i)}= & X_{n}^{\left(j_{1}\right)} X_{n}^{\left(j_{2}\right)}+\cdots+X_{n}^{\left(j_{2 b-1}\right)} X_{n}^{\left(j_{2 b}\right)} \\
& +\left(Z_{n}^{\left(j_{1}\right)} Z_{n}^{\left(j_{2}\right)}+\cdots+Z_{n}^{\left(j_{2 b-1}\right)} Z_{n}^{\left(j_{2 b}\right)}\right)^{2} \\
& -\left[\left(Z_{n}^{\left(j_{1}\right)} Z_{n}^{\left(j_{2}\right)}\right)^{2}+\cdots+\left(Z_{n}^{\left(j_{2 b-1}\right)} Z_{n}^{\left(j_{2 b}\right)}\right)^{2}\right]
\end{aligned}
$$

Then, as above, the averages of functions of $X$ and $Z$ can be calculated as in (5\&). When one only wants derivatives of $X_{n}$ at $y=0$ [see (10)], one usually iterates $Z$ and derivatives of $X$ at $y=0$ instead of doing a numerical derivation by choosing a small value of $y$.

The overlaps $q_{2}$ and $q_{3}$ obtained using this Monte Carlo method are plotted on Figs. $4 \mathrm{a}$ and $4 \mathrm{~b}$ for a hierarchical lattice of $n=16$ generations using $N=5 \times 10^{4}$ and $N=2 \times 10^{5}$. We see, as for the specific heat, a smooth 


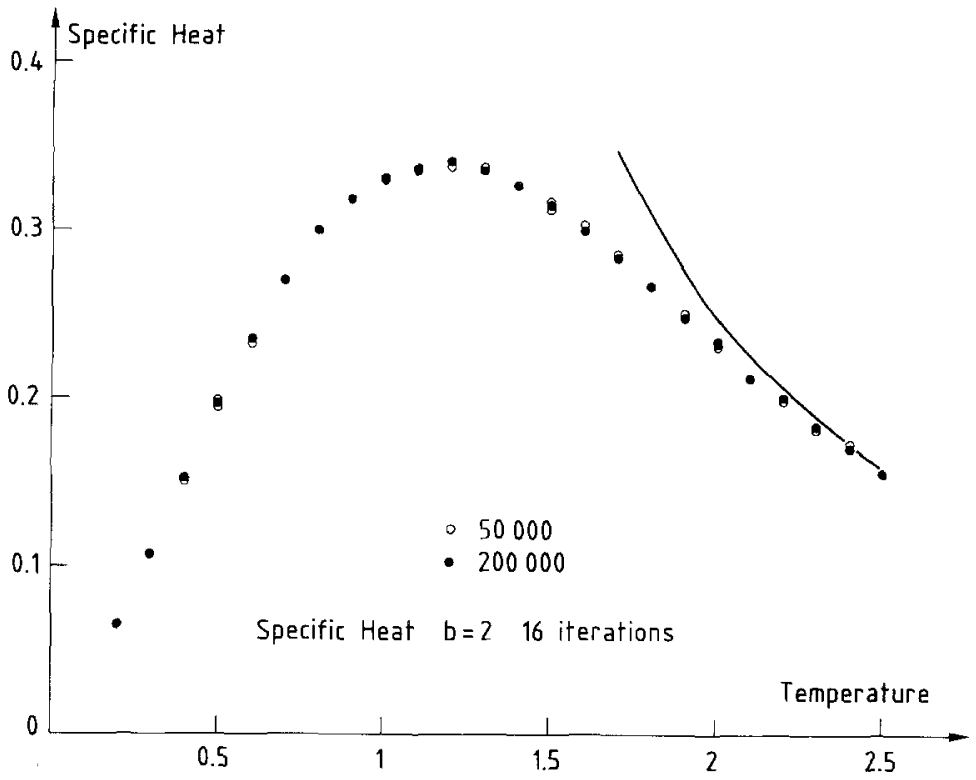

(a)

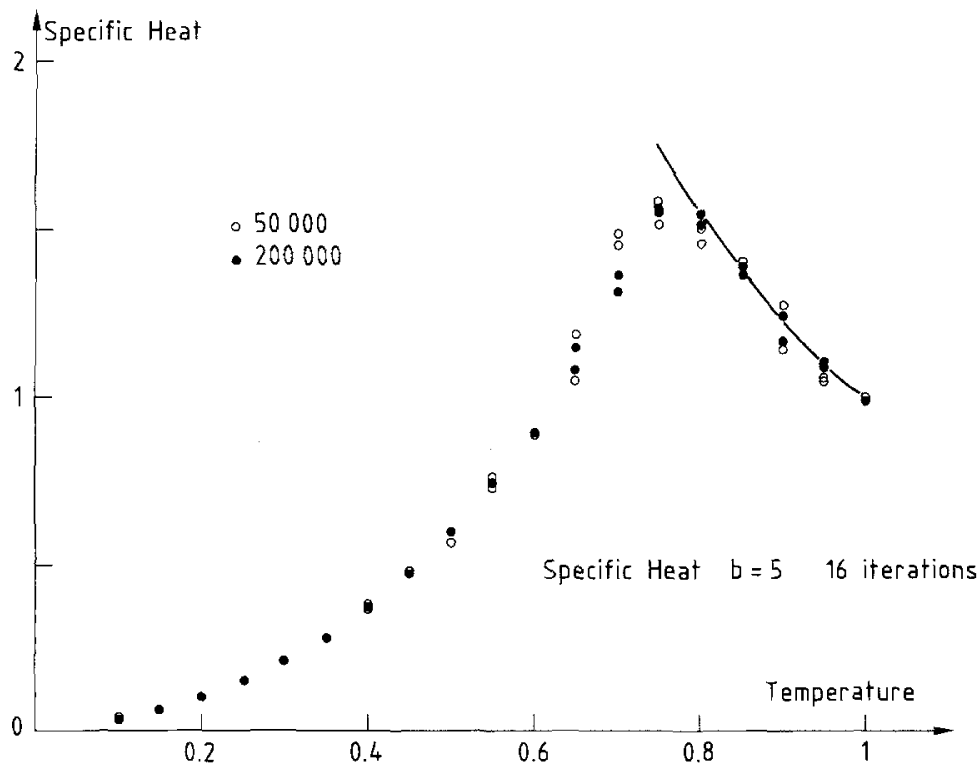

(b)

Fig. 3. The specific heat of a lattice at the 16 th generation against temperature for (a) $b=2$ and (b) $b=5$. Simulations are with sample sizes of $(0) 50,000$ and (-) 200,000. ( $\rightarrow$ ) The specific heat [Eq. (52)] of the high-temperature phase. 


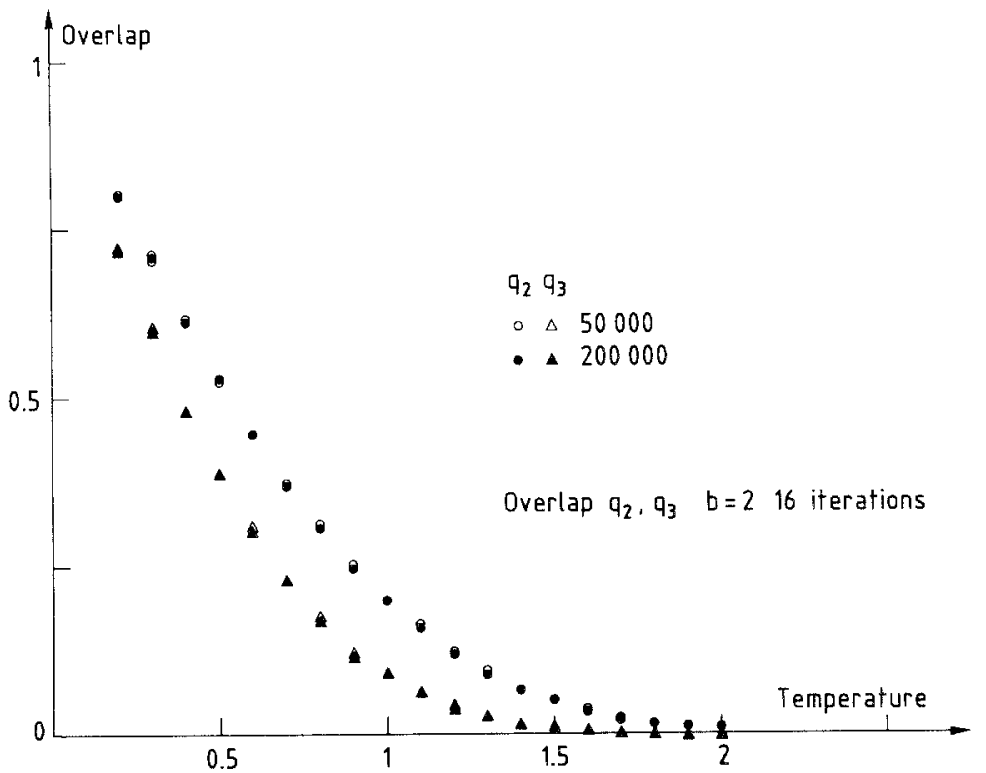

(a)

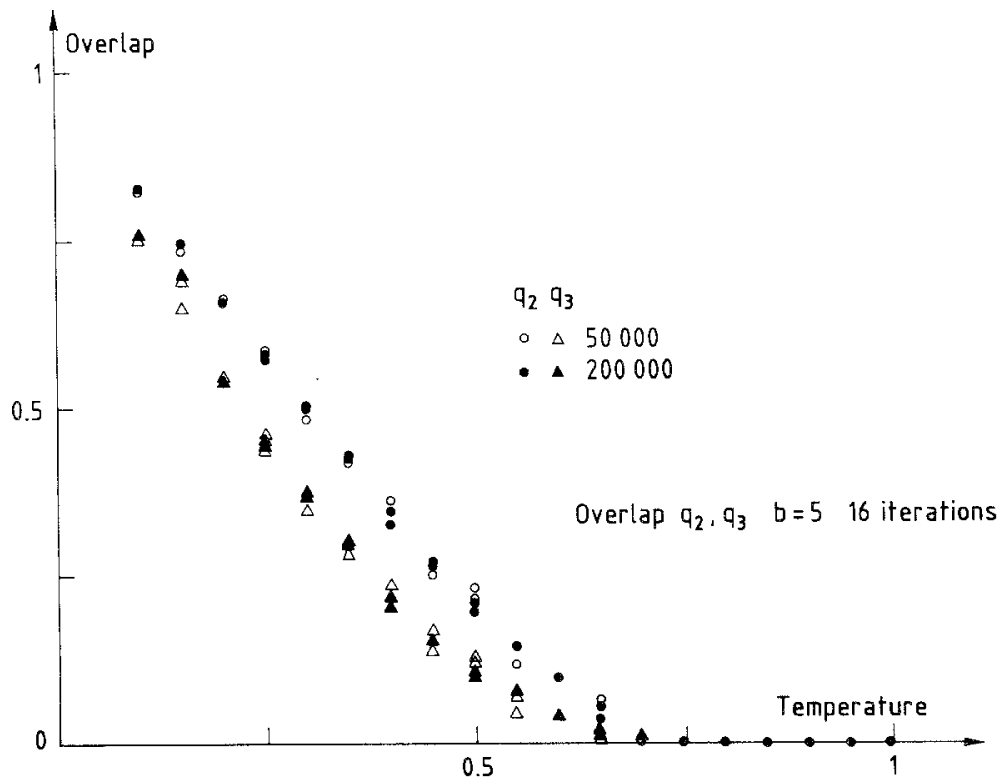

(b)

Fig. 4. The overlaps $(O, \bullet) q_{2}$ and $(\Delta, \boldsymbol{\Delta}) q_{3}$ against temperature for a lattice of 16 generations for (a) $b=2$ and (b) $b=5$. Simulations are with sample sizes of $(O, \triangle) 50,000$ and $(\bullet, \Delta) 200,000$. 
temperature dependence for $b=2$, whereas for $b=5$ both $q_{2}$ and $q_{3}$ seem to vanish at

$$
T_{c}=0.75 \pm 0.05
$$

The Monte Carlo method used here has the great advantage of being simple to implement. Its main disadvantage is that as the number $n$ of iterations increases, the $N$ values $Z_{n}^{(i)}$ become more and more correlated (because they are calculated from the same series of $Z_{n-1}^{(i)}$ instead of being independent as they should be). It is hard to estimate how the error due to these correlations depends on the number $n$ of iterations and on the number $N$ of events used to represent the distribution. It seems reasonable, however, that the larger $N$ is, the better the results are. So we usually repeated our calculation for two different large values of $N\left(N=5 \times 10^{4}\right.$ and $N=2 \times 10^{5}$ ) and we believe our results to be reliable when they are identical for these two sizes. Also, for each size $N$, we did two independent runs (which are plotted on Figs. 3 and 4 ) in order check that the fluctuations are not too large.

\section{THE FREE ENERGY AROUND $b=1$}

It was mentioned in Section 2 that when $b=1$ the problem becomes simple. This simplification can be used to provide a base for a perturbative treatment of the recursions obtained in Sections 2 and 3. In this section the perturbative approach is presented and used to derive an expression for the free energy first to first order in the small parameter $\varepsilon$, where

$$
\varepsilon=b-1
$$

and later to higher order in $\varepsilon$.

When $b=1$ the recursion (5) for the partition function reduces to

$$
Z_{n+1}=Z_{n}^{(1)} Z_{n}^{(2)}
$$

and so the average, over disorder, of $\log Z_{n}$ obeys the simple relation

$$
\left\langle\log Z_{n+1}\right\rangle=2\left\langle\log Z_{n}\right\rangle
$$

Suppose that the probability distribution of the energies $\rho\left(\varepsilon_{i j}\right)$ is given by (4). From Eq. (7), the probability distribution of $Z_{1}$ for the first generation of the lattice, $\pi_{1}(Z)$, is given by

$$
\pi_{1}(Z)=\frac{T}{(2 \pi)^{1 / 2}} \exp \left[-\frac{T^{2}}{2}(\log Z)^{2}-\log Z\right]
$$


Hence it is easy to show that the probability distribution of $Z_{n}, \pi_{n}(Z)$, at the $n$th generation of the lattice will be of the form

$$
\pi_{n}(Z)=\frac{1}{(2 \pi)^{1 / 2} \delta_{n}} \exp \left[-\frac{1}{2} \frac{(\log Z)^{2}}{\delta_{n}^{2}}-\log Z\right]
$$

where

$$
\delta_{n}=\sqrt{2^{n-1}} / T
$$

So, for $b=1$, the stable law of the recursion (5) is Gaussian in $\log Z$, as stated in Section 2.

Now returning to the case of $b>1$, we proceed to seek an expansion around $b=1$. To do this, it is helpful to write the recursion for $\left\langle\log Z_{n+1}\right\rangle$ in the following manner:

$$
\begin{aligned}
\left\langle\log Z_{n+1}\right\rangle= & \left\langle\log \left(Z_{n}^{(1)} Z_{n}^{(2)}\right)\right\rangle \\
& +\left\langle\log \left(Z_{n}^{(1)} Z_{n}^{(2)}+\cdots+Z_{n}^{(2 b-1)} Z_{n}^{(2 b)}\right)-\log \left(Z_{n}^{(1)} Z_{n}^{(2)}\right)\right\rangle
\end{aligned}
$$

where the expression occurring for $b=1$ has simply been added to and subtracted from the right-hand side.

Using the integral representation of the logarithm

$$
\log x=\int_{0}^{\infty} \frac{e^{-t}-e^{-t x}}{t} d t
$$

one can rewrite the above as

$$
\begin{aligned}
\left\langle\log Z_{n+1}\right\rangle= & 2\left\langle\log Z_{n}\right\rangle+\left\langle\int _ { 0 } ^ { \infty } \frac { d t } { t } \left\{\exp \left(-t Z_{n}^{(1)} Z_{n}^{(2)}\right)\right.\right. \\
& \left.\left.-\exp \left[-t\left(Z_{n}^{(1)} Z_{n}^{(2)}+\cdots+Z_{n}^{(2 b-1)} Z_{n}^{(2 b)}\right)\right]\right\}\right\rangle \\
= & 2\left\langle\log Z_{n}\right\rangle+\int_{0}^{\infty} \frac{d t}{t}\left[\left\langle\exp \left(-t Z_{n}^{(1)} Z_{n}^{(2)}\right)\right\rangle\right. \\
& \left.-\left\langle\exp \left(-t Z_{n}^{(1)} Z_{n}^{(2)}\right)\right\rangle^{b}\right]
\end{aligned}
$$

using the fact that the $Z_{n}^{(i)}$ are independent random variables.

It is useful to define a function $\widetilde{f}_{n}(t)$ such that

$$
\widetilde{f}_{n}(t)=\left\langle\exp \left(-t Z_{n}^{(1)} Z_{n}^{(2)}\right)\right\rangle
$$


Then the recursion, valid for all $b$, can be written as

$$
\left\langle\log Z_{n+1}\right\rangle=2\left\langle\log Z_{n}\right\rangle+\int_{0}^{\infty} \frac{d t}{t}\left\{\widetilde{f}_{n}(t)-\left[\widetilde{f}_{n}(t)\right]^{b}\right\}
$$

This can clearly be seen to reduce to the correct recursion when $b=1$. Now Eq. (65) can be expanded about $b=1$, in powers of $\varepsilon$, where

$$
\begin{aligned}
& \varepsilon=b-1 \\
&\left\langle\log Z_{n+1}\right\rangle=2\left\langle\log Z_{n}\right\rangle-\varepsilon \int_{0}^{\infty} \frac{d t}{t} \tilde{f}_{n}(t) \log \tilde{f}_{n}(t) \\
&-\frac{\varepsilon^{2}}{2} \int_{0}^{\infty} \frac{d t}{t} \tilde{f}_{n}(t) \log ^{2} \tilde{f}_{n}(t)+\cdots
\end{aligned}
$$

To obtain the recursion to first order in $\varepsilon$, it is only necessary to determine $f_{n}(t)$ to leading order, i.e., zeroth order in $\varepsilon$, or, when $b=1$

$$
\begin{aligned}
\tilde{f}_{n}(t) & =\left\langle e^{-t Z_{n}^{(1)} Z_{n}^{(2)}}\right\rangle \\
& =\left\langle e^{-t Z_{n+1}}\right\rangle+O(\varepsilon) \\
& =\int_{-\infty}^{\infty} \pi_{n+1}\left(e^{x / T}\right) \frac{1}{T} \exp \left[\frac{x}{T}-t e^{x / T}\right] d x+O(\varepsilon) \\
& =f\left(t, 2^{1 / 2} \delta_{n}\right)+O(\varepsilon)
\end{aligned}
$$

where

$$
f(t, \lambda)=\frac{1}{(2 \pi)^{1 / 2}} \int_{-\infty}^{+\infty} e^{-x^{2} / 2} e^{-t e^{i x}} d x
$$

Hence, to first order in $\varepsilon$,

$\left\langle\log Z_{n+1}\right\rangle=2\left\langle\log Z_{n}\right\rangle-\varepsilon \int_{0}^{\infty} \frac{d t}{t} f\left(t, 2^{1 / 2} \delta_{n}\right) \log f\left(t, 2^{1 / 2} \delta_{n}\right)+O\left(\varepsilon^{2}\right)$

Using this recursion it is then easy to show that, to first order in $\varepsilon$, the average free energy per unit length of the system at the $n$th generation is given by

$F_{n}=-\frac{T}{2^{n-1}}\left\langle\log Z_{n}\right\rangle=T \varepsilon \sum_{i=1}^{n-1} \frac{1}{2^{i}} \int_{0}^{\infty} f\left(t, \frac{\sqrt{2^{i}}}{T}\right) \log f\left(t, \frac{\sqrt{2^{i}}}{T}\right) \frac{d t}{t}$

It will be shown in Sections 6 and 7 that the overlaps and moments of the partition function can also be obtained to order $\varepsilon$ by the same approach and that they also can be expressed in terms of the function $f(t, \lambda)$ only. 
Having obtained the expression for the free energy $F_{n}$ to first order in $\varepsilon$, the energy $E_{n}$ and specific heat $C_{n}$ of the system at the $n$th generation can be obtained to the same order by differentiating $F_{n}$ with respect to temperature $T$. The specific heat obtained in this manner is shown as a function of temperature in Fig. 5 for a lattice of $n=8$ generations. We see that the shape (linear at low temperature and the maximum at $T \simeq 1.0$ ) is rather similar to the results of the Monte Carlo method for $b=2$.

At low temperature, using the asymptotic forms of $f(t, \lambda)$ for $\lambda \rightarrow \infty$, it is possible [see Appendix A, Eq. (A16)] to simplify the expression for the free energy to obtain

$$
\begin{aligned}
F_{n}= & \varepsilon\left\{-K_{1} \sum_{i=1}^{n-1} \frac{1}{\sqrt{2^{i}}}+\frac{1}{2}\left[\left(\Gamma^{(1)}(1)\right)^{2}(1)-\Gamma^{(2)}(1)\right] K_{1} T^{2} \sum_{i=1}^{n-1} \frac{1}{2^{3 i / 2}}\right. \\
& \left.+K_{2} T^{3}\left[\Gamma^{(1)}(1) \Gamma^{(2)}(1)-\frac{2}{3}\left(\Gamma^{(1)}(1)\right)^{3}-\frac{\Gamma^{(3)}(1)}{3}\right]_{i=1}^{n-1} \frac{1}{4^{i}}+O\left(T^{4}\right)\right\}
\end{aligned}
$$

where

$$
\begin{aligned}
& K_{1}=-\int \frac{t e^{-t^{2} / 2}}{(2 \pi)^{1 / 2}} \log \left[\int_{t}^{\infty} e^{-u^{2} / 2} \frac{d u}{(2 \pi)^{1 / 2}}\right] d t=0.90320 \\
& K_{2}=\int\left(\frac{1-t^{2}}{2}\right) \frac{e^{-t^{2} / 2}}{(2 \pi)^{1 / 2}} \log \left[\int_{t}^{\infty} e^{-u^{2} / 2} \frac{d u}{(2 \pi)^{1 / 2}}\right] d t=0.29782
\end{aligned}
$$

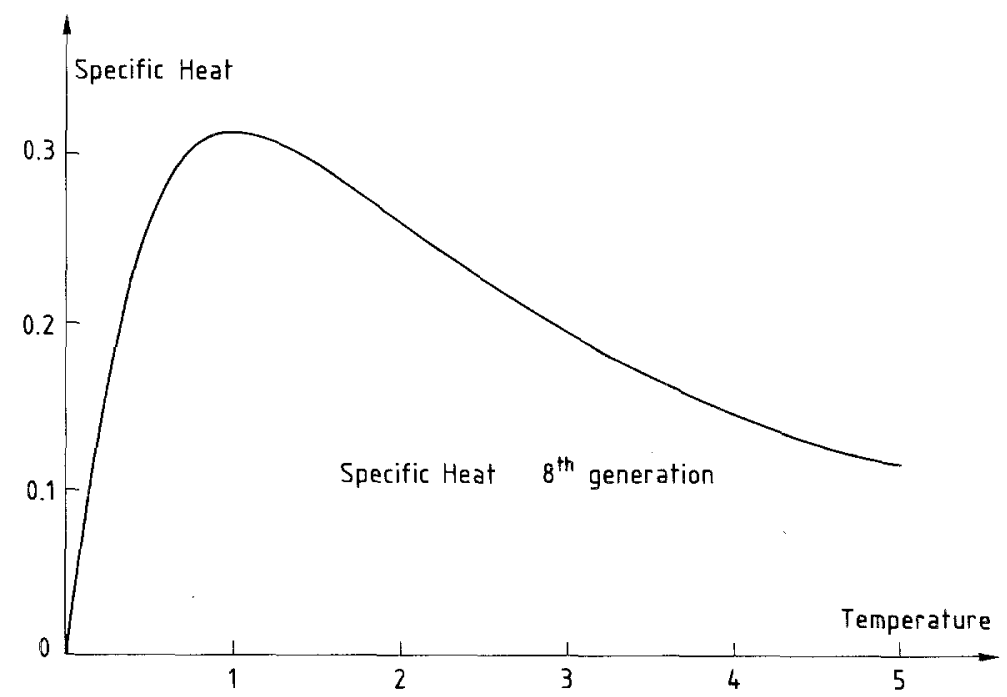

Fig. 5. The specific heat of a lattice of eight generations calculated from the perturbative expansion [Eq. (70)]. It looks rather similar to the results for $b=2$ shown in Fig. 3a. 
In the limit that $T$ tends to zero the ground-state energy obtained in ref. 23 is recovered.

In the high-temperature expansion, it is not possible to get an analytic expression for the coefficients. The reason is that the infinite-temperature fixed point is an unstable one $\left(\delta_{n+1}=2^{1 / 2} \delta_{n}\right)$ and therefore the values of some coefficients of the high-temperature expansion depend on the functions at all points $\delta_{n}, \delta_{n}$ small, $n \ll(\log T) / \log \sqrt{2}, \delta_{n}$ large, $n \gg(\log T) / \log \sqrt{2}$, and also intermediate values $\delta_{n}$ where no analytic expression can be obtained. Nevertheless, from Eq. (70) one expects the leading behavior of $F_{n}$ at high $T$ to be

$$
\lim _{n \rightarrow \infty} F_{n} \sim-\varepsilon T
$$

In the remainder of this section we will show how the perturbative approach can be extended to higher order in $\varepsilon$. To do this, it is necessary to determine the function $\tilde{f}_{n}(t)$ defined by $(64)$ to higher order in $\varepsilon$. Below we show how to obtain the first correction to $f_{n}(t)$.

Let us define a new function $\widetilde{F}_{n}(t)$ by

$$
\tilde{F}_{n}(t)=\left\langle e^{-t Z_{n}}\right\rangle
$$

Then, from (64), (75), and (5) one has, for all $b$, that

$$
\tilde{F}_{n+1}(t)=\left[\tilde{f}_{n}(t)\right]^{b}
$$

Hence, $\tilde{F}_{n+1}(t)$ is related to $\tilde{f}_{n}(t)$. Now we need to find another relation to obtain $\tilde{f}_{n}(t)$ from $\tilde{F}_{n}(t)$. We could not do this for arbitrary $b$. However, for $b$ close to 1 , one can consider that the probability distribution $R_{n}(Y)$ of the quantity $Y=\log Z_{n}$ is the sum of a Gaussian distribution and a small correction,

$$
R_{n}(Y)=\frac{1}{(2 \pi)^{1 / 2} \delta_{n}} \exp \left(\frac{-Y^{2}}{2 \delta_{n}^{2}}\right)+\varepsilon \frac{1}{\delta_{n}} \varphi_{n}\left(\frac{Y}{\delta_{n}}\right)
$$

It is then clear that the probability distribution $r_{n}(y)$ of the quantity $y=\log \left(Z_{n}^{(1)} Z_{n}^{(2)}\right)$ is given by

$$
\begin{aligned}
r_{n}(y)= & \int R_{n}(Y) R_{n}(y-Y) d Y \\
= & \frac{1}{(2 \pi)^{1 / 2} 2^{1 / 2} \delta_{n}} \exp \left(-\frac{y^{2}}{4 \delta_{n}^{2}}\right) \\
& +\frac{2 \varepsilon}{(2 \pi)^{1 / 2} \delta_{n}^{2}} \int \varphi_{n}\left(\frac{Y}{\delta_{n}}\right) e^{-(Y-y)^{2} / 2 \delta_{n}^{2}} d Y+O\left(\varepsilon^{2}\right)
\end{aligned}
$$

From Eq. (75) and (77), $\widetilde{F}_{n}(t)$ can then be expressed as

$$
\tilde{F}_{n}(t)=\int R_{n}(Y) e^{-t e^{Y}} d Y=f\left(t, \delta_{n}\right)+\varepsilon G_{n}(t)
$$


where

$$
G_{n}(t)=\int \varphi_{n}(Y) \exp \left(-t e^{\delta_{n} Y}\right) d Y
$$

Similarly, from Eqs. (64) and (78), we see that $\tilde{f}_{n}(t)$ is given for $b$ close to 1 by

$$
\begin{aligned}
\widetilde{f}_{n}(t) & =\int r_{n}(y) e^{-t e^{y}} d y \\
& =f\left(t, 2^{1 / 2} \delta_{n}\right)+2 \varepsilon \int \frac{e^{-y^{2} / 2}}{(2 \pi)^{1 / 2}} G_{n}\left(t e^{\delta_{n} y}\right) d y
\end{aligned}
$$

Now, using the relation between $\tilde{f}_{n}(t)$ and $\tilde{F}_{n+1}(t)$ of Eq. (76) and expanding it for $b=1+\varepsilon$, one can find a recursion for $G_{n}(t)$, which reads

$$
G_{n+1}(t)=f\left(t, 2^{1 / 2} \delta_{n}\right) \log f\left(t, 2^{1 / 2} \delta_{n}\right)+2 \int \frac{e^{-y^{2} / 2}}{(2 \pi)^{1 / 2}} G_{n}\left(t e^{\delta_{n} y}\right) d y
$$

Carrying out this recursion, with the initial condition that $\varphi_{1}(Y)=0, G_{n}(t)$ can be computed explicitly and we find from Eq. (81) that

$$
\begin{aligned}
\widetilde{f}_{n}(t)= & f\left(t, 2^{1 / 2} \delta_{n}\right)+\varepsilon \int_{-\infty}^{\infty} \sum_{i=1}^{n-1} 2^{n-i} f\left(t \exp \left[\frac{\left(2^{n}-2^{i}\right)^{1 / 2} y}{T}\right], \frac{\sqrt{2^{i}}}{T}\right) \\
& \times \log f\left(t \exp \left[\frac{\left(2^{n}-2^{i}\right)^{1 / 2} y}{T}\right], \frac{\sqrt{2^{i}}}{T}\right) \frac{\exp \left(-y^{2} / 2\right)}{(2 \pi)^{1 / 2}} d y+O\left(\varepsilon^{2}\right)
\end{aligned}
$$

Having determined $\tilde{f}_{n}(t)$ to first order in $\varepsilon$, it is now possible to use Eq. (66) to calculate the free energy, and indeed the overlaps and moments to be discussed in the next sections, to second order in $\varepsilon$. For example, the free energy now becomes

$$
\begin{aligned}
F_{n}(T)= & \varepsilon T \sum_{i=1}^{n-1} \frac{1}{2^{i}}\left[\int_{0}^{\infty} \frac{d t}{t} f\left(t, \frac{\sqrt{2^{i}}}{T}\right) \log f\left(t, \frac{\sqrt{2^{i}}}{T}\right)\right. \\
& +\frac{\varepsilon}{2} \int_{0}^{\infty} \frac{d t}{t} f\left(t, \frac{\sqrt{2^{i}}}{T}\right) \log ^{2} f\left(t, \frac{\sqrt{2^{i}}}{T}\right) \\
& +\varepsilon \int_{0}^{\infty} \frac{d t}{t}\left[1+\log f\left(t, \frac{\sqrt{2^{i}}}{T}\right)\right] \sum_{j=1}^{i-1} 2^{i-j} \\
& \times \int_{0}^{\infty} f\left(t \exp \left[\frac{\left(2^{i}-2^{j}\right)^{1 / 2} y}{T}\right], \frac{\sqrt{2^{j}}}{T}\right) \\
& \left.\times \log f\left(t \exp \left[\frac{\left(2^{i}-2^{j}\right)^{1 / 2} y}{T}\right], \frac{\sqrt{2^{j}}}{T}\right) \frac{\exp \left(-y^{2} / 2\right) d t}{(2 \pi)^{1 / 2}}\right] \\
& +O\left(\varepsilon^{3}\right)
\end{aligned}
$$




\section{PERTURBATIVE TREATMENT OF OVERLAPS}

Following the same approach as in the previous section, expressions for the overlaps will now be obtained to first order in $\varepsilon$. The lowtemperature behavior of these quantities will then be considered.

First let us consider the average overlap between two walks, $\left\langle q_{2}\left(W, W^{\prime}\right)\right\rangle$. As discussed in Section 2, it is convenient when dealing with this problem to use the generating function $X_{n}(T, y)$ defined in (10). These generating functions were shown in Section 2 to obey the recursion given in Eq. (11). When $b=1$ this recursion reduces to a simple expression:

$$
X_{n+1}(T, y)=X_{n}(T, y)^{(1)} X_{n}(T, y)^{(2)}
$$

Using the same procedure as in Section 5, we consider the quantity $\phi_{n}(v+2)=\left\langle X_{n}(T, y) Z_{n}^{v}\right\rangle$. For an arbitrary power $v$, the following recursion can then be written as

$$
\begin{aligned}
\left\langle X_{n+1}\right. & \left.Z_{n+1}^{v}\right\rangle \\
= & \left\langle X_{n}^{(1)} X_{n}^{(2)}\left(Z_{n}^{(1)} Z_{n}^{(2)}\right)^{v}\right\rangle \\
& +\left\langle\left(X_{n}^{(1)} X_{n}^{(2)}+\cdots+X_{n}^{(2 b-1)} X_{n}^{(2 b)}\right)\right. \\
& \left.\times\left(Z_{n}^{(1)} Z_{n}^{(2)}+\cdots+Z_{n}^{(2 b-1)} Z_{n}^{(2 b)}\right)^{v}\right\rangle-\left\langle X_{n}^{(1)} X_{n}^{(2)}\left(Z_{n}^{(1)} Z_{n}^{(2)}\right)^{v}\right\rangle \\
& +\left\langle\left(Z_{n}^{(1)} Z_{n}^{(2)}+\cdots+Z_{n}^{(b-1)} Z_{n}^{(2 b)}\right)^{v+2}\right\rangle-\left\langle\left(Z_{n}^{(1)} Z_{n}^{(2)}\right)^{v+2}\right\rangle \\
& -\left\langle\left[\left(Z_{n}^{(1)} Z_{n}^{(2)}\right)^{2}+\cdots+\left(Z_{n}^{(2 b-1)} Z_{n}^{(2 b)}\right)^{2}\right]\right. \\
& \left.\times\left(Z_{n}^{(1)} Z_{n}^{(2)}+\cdots+Z_{n}^{(2 b-1)} Z_{n}^{(2 b)}\right)^{v}\right\rangle+\left\langle\left(Z_{n}^{(1)} Z_{n}^{(2)}\right)^{v+2}\right\rangle
\end{aligned}
$$

Following the spirit of the previous calculation, we introduce the function $\tilde{g}_{y, n}\left(t, t^{\prime}\right)$ defined as

$$
\tilde{g}_{y, n}\left(t, t^{\prime}\right)=\left\langle\exp \left(-t Z_{n}^{(1)} Z_{n}^{(2)}\right) \exp \left[-t X_{n}^{(1)}(T, y) X_{n}^{(2)}(T, y)\right]\right\rangle
$$

and we appeal to the following integral representations of $Z^{v}$ to help linearize the problem:

$$
\begin{aligned}
& Z^{v}=\int_{0}^{\infty} \frac{d t t^{-v-1}}{\Gamma(-v)} e^{-t Z} \quad v<0 \\
& Z^{v}=\int_{0}^{\infty} \frac{d t t^{p-v-1}}{\Gamma(p-v)}(-1)^{p} \frac{d^{p}}{d t^{p}}\left[e^{-t Z}\right] \\
& v>0 \text { and } \quad p>v, \quad p \text { integer }
\end{aligned}
$$

(For clarity the case $v=-2$ will be considered first and the results for $v>0$, which follow in exactly the same manner, will be given at the end. 
$-2<v<0$ can be considered in the same way.) Equation (86) can then be rewritten in the form

$$
\begin{aligned}
\left\langle X_{n+1} Z_{n+1}^{v}\right\rangle= & \left\langle X_{n} Z_{n}^{v}\right\rangle^{2}-\int_{0}^{\infty} \frac{d t t^{-v-1}}{\Gamma(-v)} \\
& \times \frac{d}{d t^{\prime}}\left\{\left[\tilde{g}_{y, n}\left(t, t^{\prime}\right)\right]^{b}-\tilde{g}_{y, n}\left(t, t^{\prime}\right)\right\}_{t^{\prime}=0} \\
& +\int_{0}^{\infty} \frac{d t t^{-v-3}}{\Gamma(-v-2)}\left\{\left[\tilde{f}_{n}(t)\right]^{b}-\tilde{f}_{n}(t)\right\} \\
& +\int_{0}^{\infty} \frac{d t t^{-v-1}}{\Gamma(-v)} \frac{d}{d t^{\prime}}\left\{\left[\tilde{g}_{0, n}\left(t, t^{\prime}\right)\right]^{b}-\tilde{g}_{0, n}\left(t, t^{\prime}\right)\right\}_{t^{\prime}=0}
\end{aligned}
$$

where $f_{n}(t)$ is as defined in Eq. (64), and we have used the fact that $X_{n}(T, 0)=Z_{n}^{2}$. It is now simple to expand about $b=1$. Again, to obtain an expression correct to first order in $\varepsilon$, the functions $\tilde{f}_{n}(t)$ and $\tilde{g}_{y, n}\left(t, t^{\prime}\right)$ will only be required at $b=1$. To this order $\tilde{f}_{n}(t)=f\left(t, \sqrt{2^{n}} / T\right)$, as shown earlier [Eq. (67)], and

since

$$
\left.\frac{d}{d t^{\prime}} \tilde{g}_{y, n}\left(t, t^{\prime}\right)\right|_{t^{\prime}=0}=-\exp \left(2^{n} y\right) \frac{d^{2} f}{d t^{2}}\left(t, \frac{\sqrt{2^{n}}}{T}\right)
$$

$$
\begin{aligned}
& \left.\frac{d}{d t^{\prime}} \tilde{g}_{y, n}\left(t, t^{\prime}\right)\right|_{t^{\prime}=0} \\
& =-\left\langle X_{n}^{(1)}(T, y) X_{n}^{(2)}(T, y) \exp \left(-t Z_{n}^{(1)} Z_{n}^{(2)}\right)\right\rangle \\
& =-\left\langle\left[\exp \left(2^{n} y\right)\right]\left(Z_{n}^{(1)} Z_{n}^{(2)}\right)^{2} \exp \left(-t Z_{n}^{(1)} Z_{n}^{(2)}\right)\right\rangle
\end{aligned}
$$

Hence Eqs. (89) reduces to

$$
\begin{aligned}
\left\langle X_{n+1} Z_{n+1}^{v}\right\rangle= & \left\langle X_{n} Z_{n}^{v}\right\rangle^{2}-\varepsilon\left(1-e^{2^{n} y}\right) \int_{0}^{\infty} \frac{d t t^{-v-1}}{\Gamma(-v)} \\
& \times \frac{d^{2} f}{d t^{2}}\left(t, \frac{\sqrt{2^{n}}}{T}\right)\left[1+\log f\left(t, \frac{\sqrt{2^{n}}}{T}\right)\right] \\
& +\varepsilon \int_{0}^{\infty} \frac{d t t^{-v-3}}{\Gamma(-v-2)} f\left(t, \frac{\sqrt{2^{n}}}{T}\right) \log f\left(t, \frac{\sqrt{2^{n}}}{T}\right)
\end{aligned}
$$

Carrying out this recursion to first order in $\varepsilon$, we obtain an expression for $\left\langle X_{n} Z_{n}^{v}\right\rangle$ in terms of the initial condition

$$
\left\langle X_{1} Z_{1}^{v}\right\rangle=(\exp y)\left\langle Z_{1}^{v+2}\right\rangle=\exp \left[y+\frac{(v+2)^{2}}{2 T^{2}}\right]
$$


which reads

$$
\begin{aligned}
& \frac{1}{2^{n-1}} \log \left\langle X_{n} Z_{n}^{v}\right\rangle \\
&=y+\frac{(v+2)^{2}}{2 T^{2}}-\varepsilon \sum_{i=1}^{n-1} \frac{1}{2^{i}} \exp \left[\frac{-(v+2)^{2}}{2} \delta_{i+1}^{2}\right] \\
& \times\left\{\left[\exp \left(-2^{i} y\right)-1\right] \int \frac{d t t^{-v-1}}{\Gamma(-v)}\right. \\
& \quad \cdot \frac{d^{2} f\left(t, \delta_{i+1}\right)}{d t^{2}}\left[1+\log f\left(t, \delta_{i+1}\right)\right] \\
&\left.-\left[\exp \left(-2^{i} y\right)\right] \int \frac{d t t^{-v-3}}{\Gamma(-v-2)} f\left(t, \delta_{i+1}\right) \log f\left(t, \delta_{i+1}\right)\right\}
\end{aligned}
$$

For $v>0$ and $p>v$, it can be shown that a similar relation holds:

$$
\begin{aligned}
\frac{1}{2^{n-1}} & \log \left\langle X_{n} Z_{n}^{v}\right\rangle \\
= & y+\frac{(v+2)^{2}}{2 T^{2}}-\varepsilon \sum_{i=1}^{n-1} \frac{1}{2^{i}} \exp \left[\frac{-(v+2)^{2}}{2} \delta_{i+1}^{2}\right] \\
& \times\left\{\left[\exp \left(-2^{i} y\right)-1\right] \int \frac{d t t^{p-v-1}}{\Gamma(p-v)}(-1)^{p}\right. \\
& \times \frac{d^{p}}{d t^{p}}\left[\frac{d^{2} f\left(t, \delta_{i+1}\right)}{d t^{2}}\left[1+\log f\left(t, \delta_{i+1}\right)\right]\right] \\
& \left.-\left[\exp \left(-2^{i} y\right)\right] \int \frac{d t t^{p-v-1}}{\Gamma(p-v)}(-1)^{p} \frac{d^{p+2}}{d t^{p+2}}\left[f\left(t, \delta_{i+1}\right) \log f\left(t, \delta_{i+1}\right)\right]\right\}
\end{aligned}
$$

The overlap $\left\langle q_{2}\left(W, W^{\prime}\right)\right\rangle$ can now be obtained by setting $v=-2$ in Eq. (94) and differentiating with respect to $y$, to yield

$$
\left\langle q_{2}\right\rangle=1+\varepsilon \sum_{i=1}^{n-1} \int_{0}^{\infty} t d t\left[1+\log f\left(t, \frac{\sqrt{2^{i}}}{T}\right)\right] \frac{d^{2} f}{d t^{2}}\left(t, \frac{\sqrt{2^{i}}}{T}\right)
$$

and the overlap of two walks calculated for $p+2$ replicas $(p>0)$ follows from Eq. (95), giving 


$$
\begin{aligned}
\left\langle Q_{n}(p+2)\right\rangle= & 1+\varepsilon \exp \left[-(p+2)^{2} \frac{2^{i-1}}{T^{2}}\right](-1)^{p} \\
& \times \sum_{i=1}^{n-1} \frac{d^{p}}{d t^{p}}\left\{\frac{d^{2} f}{d t^{2}}\left(t, \frac{\sqrt{2^{i}}}{T}\right)\left[1+\log f\left(t, \frac{\sqrt{2^{i}}}{T}\right)\right]\right. \\
& \left.-\frac{d^{2}}{d t^{2}}\left[f\left(t, \frac{\sqrt{2^{i}}}{T}\right) \log f\left(t, \frac{\sqrt{2^{i}}}{T}\right)\right]\right\}\left.\right|_{t=0}
\end{aligned}
$$

As mentioned in Section 2, it is possible, using Eqs. (14), (16), and (88a), to compute in the same way the average overlap between $m$ walks, $\left\langle q_{m}\right\rangle$, giving

$$
\left\langle q_{m}\right\rangle=1+\varepsilon(-1)^{m} \sum_{i=1}^{n-1} \int \frac{d t}{\Gamma(m)}\left[1+\log f\left(t, \frac{\sqrt{2^{i}}}{T}\right)\right] \frac{d^{m} f\left(t, \sqrt{2^{i}} / T\right)}{d t^{m}}
$$

It is possible to obtain the low-temperature expansions of these three overlaps, (96)-(98). Using the appropriate expansion of $f(t, \lambda)$, as explained in Appendix A, it can be shown that the first correction to $\left\langle q_{2}\right\rangle$ is linear in temperature

$$
\left\langle q_{2}\right\rangle=1-\varepsilon K_{1} T \sum_{i=1}^{n-1}-\frac{1}{\sqrt{2^{i}}}+O\left(T^{3}\right)
$$

where $K_{1}$ is defined in Eq. (72). A similar expression can be obtained for $\left\langle q_{m}\right\rangle$,

$$
\begin{aligned}
\left\langle q_{m}\right\rangle= & 1-\varepsilon K_{1} T\left(\frac{\Gamma^{\prime}(m)}{\Gamma(m)}-\Gamma^{\prime}(1)\right)_{i=1}^{n-1} \frac{1}{\sqrt{2^{i}}} \\
& -\varepsilon K_{2} T^{2}\left[\frac{\Gamma^{\prime \prime}(m)}{\Gamma(m)}-\frac{2 \Gamma^{\prime}(1) \Gamma^{\prime}(m)}{\Gamma(m)}+2 \Gamma^{\prime}(1)^{2}-\Gamma^{\prime \prime}(1)\right]_{i=1}^{n-1} \frac{1}{2^{i}}+O\left(T^{3}\right)
\end{aligned}
$$

It is interesting to compare these results with the expressions of the overlaps $q_{m}$ on the tree. ${ }^{(1)}$ For the tree, it would be possible to show (but we will not here) that the overlaps $\left\langle q_{m}\right\rangle$ are given by $\left\langle q_{m}\right\rangle=$ $\Gamma\left(m-T / T_{c}\right) / \Gamma(m) \Gamma\left(1-T / T_{c}\right)$. At low temperature, this gives the same expression as (100) with $\varepsilon K_{1} \sum 1 / \sqrt{2^{i}}$ replaced by $1 / T_{c}$ and $\varepsilon K_{2} \sum 1 / 2^{i}$ by $-1 / 2 T_{c}^{2}$.

To calculate $Q_{n}(p+2)$, it is necessary to know the first $p+1$ derivatives of $f\left(t, \sqrt{2^{i}} T\right)$ with respect to $t$ at $t=0$. However, as these derivatives have the simple form

$$
f^{(p)}\left(0, \frac{\sqrt{2^{i}}}{T}\right)=(-1)^{p} \exp \frac{2^{i-1} p^{2}}{T^{2}}
$$


the leading term at low temperature can be seen to result from taking $i=1$ and the term with the highest possible derivative of $f\left(t, 2^{1 / 2} / T\right)$. Hence, it follows that

$$
\begin{aligned}
Q_{n}(2) & =1-\varepsilon e^{-2 / T^{2}}+\cdots \\
Q_{n}(2+p) & =1-2 \varepsilon e^{-2(p+1) / T^{2}}+\cdots, \quad p \geqslant 1
\end{aligned}
$$

It can be seen that $Q_{n}(2)$ obtained in this manner agrees with that resulting from Eqs. (46)-(48) expanded for $b=1+\varepsilon$, as expected. The first corrections to $Q_{n}(2+p)$ are all exponentially small in temperature. We see from (99), (102), and (103) that the first-order correction to the overlap $Q_{n}(2+v)$ is exponentially small in the temperature except for $v=-2$, when it vanishes linearly with temperature. Similar behavior will be seen in the next section when the moments of the partition function are considered.

As the polymer problem has many analogies with spin glasses, it is interesting to consider the distribution of overlaps $P_{2}(q)$ defined in Eq. (9). In the Parisi theory of infinite-range spin glasses the corresponding quantity, $P(q)$, is a broad distribution, even in the thermodynamic limit. ${ }^{(29-31)}$ It has been debated a great deal whether this property of $P(q)$ persists in finite-dimensional spin glasses. So it is interesting to see whether $P_{2}(q)$ possesses this spin-glass-like property.

To determine the shape of the distribution $P_{2}(q)$, let us define $y_{n}$ by

$$
e^{y_{n}}=\left\langle X_{n}(T, y) Z_{n}^{-2}\right\rangle
$$

Then, from recursion (94) the following recursion for $y_{n}$ can be obtained:

where

$$
y_{n+1}=2^{n} y_{1}+\varepsilon \sum_{i=1}^{n} 2^{n-i} A_{i}\left(1-e^{-2 y_{i}}\right)
$$

$$
A_{i}=\int_{0}^{\infty} d t t \frac{d^{2} f}{d t^{2}}\left(t, \frac{\sqrt{2^{i}}}{T}\right)\left[1+\log f\left(t, \frac{\sqrt{2^{i}}}{T}\right)\right]
$$

Using a renormalized variable $\tilde{y}$ given by

$$
\tilde{y}=2^{n} y_{1}
$$

this can be recast as

$$
\begin{aligned}
y_{n+1} & =\tilde{y}+\varepsilon \sum_{i=1}^{n} 2^{n-i}\left[1-\exp \left(-2^{i-n} \tilde{y}\right)\right] A_{i} \\
& \rightarrow \tilde{y}\left(1+\varepsilon \sum_{i=1}^{\infty} A_{i}\right) \quad \text { as } \quad n \rightarrow \infty
\end{aligned}
$$


where we have taken the limit $n \rightarrow \infty$ and noticed that, as $A_{i} \rightarrow 0$ when $i \rightarrow \infty$, only terms with $n-i \gg 0$ will contribute to the sum.

Hence we see that

$$
\left\langle X_{n}(T, y) Z_{n}^{-2}\right\rangle=\exp \left[\tilde{y}\left(1+\varepsilon \sum_{i=1}^{n} A_{i}\right)\right]
$$

This shows that, at least to first order in $\varepsilon, P_{2}(q)$ is a delta function at $\left\langle q_{2}\right\rangle$. For the problem of polymers on a disordered tree (mean field model $\left.{ }^{(1)}\right)$, it has been found that $P_{2}(q)$ does have a broad distribution (more precisely, it is the sum of two delta functions at $q=0$ and $q=1$ ). Since one expects that, when $b$ becomes large, the model on the hierarchical lattice would behave like a mean-field model, it raises the question of whether there is a critical value $b_{c}$ of $b$ above which the mean-field features appear [i.e., $P_{2}(q)$ becomes broad] or whether the mean-field behavior is limited to $b=\infty$.

\section{NONINTEGER MOMENTS AND REPLICA}

We now turn to a consideration of the moments of the partition function. First, an expression will be obtained for an arbitrary moment $\left\langle Z_{n}^{v}\right\rangle$, to first order in $\varepsilon$, and then the low-temperature limit will be considered.

Consider, then, the average of the $v$ th power of the partition function at the $n$th generation of the lattice, $\left\langle Z_{n}^{v}\right\rangle$ (take $v<0$ to begin with). Following the perturbative approach of $(61)$ and (86), we can write the recursion for this quantity

$$
\begin{aligned}
\left\langle Z_{n+1}^{v}\right\rangle & =\left\langle Z_{n}^{v}\right\rangle^{2}+\left[\left\langle\left(Z_{n}^{(1)} Z_{n}^{(2)}+\cdots+Z_{n}^{(2 b-1)} Z_{n}^{(2 b)}\right)^{v}\right\rangle-\left\langle\left(Z_{n}^{(1)} Z_{n}^{(2)}\right)^{v}\right\rangle\right] \\
& =\left\langle Z_{n}^{v}\right\rangle^{2}+\left[\int_{0}^{\infty} \frac{d t t^{-v-1}}{\Gamma(-v)}\left\{\left[\tilde{f}_{n}(t)\right]^{b}-f_{n}(t)\right\}\right]
\end{aligned}
$$

where we have used the integral representation of Eq. (88a) and the usual function $\tilde{f}_{n}(t)$ defined in Eq. (64).

Expanding now, to first order in $\varepsilon$, we obtain

$$
\left\langle Z_{n+1}^{v}\right\rangle=\left\langle Z_{n}^{v}\right\rangle^{2}+\frac{\varepsilon}{\Gamma(-v)} \int_{0}^{\infty} d t t^{-\nu-1} f\left(t, \frac{\sqrt{2^{n}}}{T}\right) \log f\left(t, \frac{\sqrt{2^{n}}}{T}\right)
$$

and so, using the initial condition

$$
\left\langle Z_{1}^{v}\right\rangle^{2}=\exp \left(v^{2} / T^{2}\right)
$$


it can be shown that for $v<0$

$$
\begin{aligned}
\frac{1}{2^{n-1}} \log \left\langle Z_{n}^{v}\right\rangle & \\
= & \frac{v^{2}}{2 T^{2}}+\varepsilon \sum_{i=1}^{n-1} \frac{1}{2^{i}} \exp \left(\frac{-v^{2} 2^{i-1}}{T^{2}}\right) \\
& \times \int_{0}^{\infty} d t \frac{t^{-v-1}}{\Gamma(-v)} f\left(t, \frac{\sqrt{2^{i}}}{T}\right) \log f\left(t, \frac{\sqrt{2^{i}}}{T}\right)
\end{aligned}
$$

For $v>0$ we have to use the alternative integral representation, Eq. (88b), and then similar expressions can be found for $v>0$, i.e., for $p>v$

$$
\begin{aligned}
\frac{1}{2^{n-1}} & \log \left\langle Z_{n}^{v}\right\rangle \\
= & \frac{v^{2}}{2 T^{2}}+\varepsilon \sum_{i=1}^{n-1} \frac{1}{2^{i}} \exp \left(\frac{-v^{2} 2^{i-1}}{T^{2}}\right) \\
& \times \int_{0}^{\infty} \frac{d t t^{p-v-1}}{\Gamma(p-v)}(-1)^{p} \frac{d^{p}}{d t^{p}}\left[f\left(t, \frac{\sqrt{2^{i}}}{T}\right) \log f\left(t, \frac{\sqrt{2^{i}}}{T}\right)\right]
\end{aligned}
$$

It is interesting to note that once again the first correction depends only on the function $f(t, \lambda)$, as did the overlaps and free energy.

For integer moments, the latter expression reduces to

$$
\begin{aligned}
\frac{1}{2^{n-1}} \log \left\langle Z_{n}^{p}\right\rangle & \\
= & \frac{p^{2}}{2 T^{2}}+\varepsilon \sum_{i=1}^{n-1} \frac{1}{2^{i}} \\
& \times\left.\exp \left(-p^{2} \frac{2^{i-1}}{T^{2}}\right)(-1)^{p} \frac{d^{p}}{d t^{p}}\left[f\left(t, \frac{\sqrt{2^{i}}}{T}\right) \log f\left(t, \frac{\sqrt{2^{i}}}{T}\right)\right]\right|_{t=0}
\end{aligned}
$$

The calculation of the derivatives of $f(t, \lambda) \log f(t, \lambda)$ with respect to $t$ at $t=0$ can be done easily [see Appendix A, (A3)] and then the above equation gives answers in agreement with those obtained from an expansion about $b=1$ of the recursions of the integer moments in (22) and (25). However, the perturbative expansion ( $\varepsilon$ small $)$ has also given the interpolation between integer moments.

It is interesting to consider whether a similar form of interpolation could be used in other problems where only the integer moments of the 
partition function can be determined. Consider a sequence of numbers $a(p)$ $(p=0,1,2, \ldots)$. The problem is how to extend them to a function $a(v)$ defined at all values of $v$. By comparing Eqs. (114) and (115), we see that the interpolation formula valid here is to construct a function $J(t)$ which plays the role of $f(t, \lambda) \log f(t, \lambda)$, via

$$
J(t)=\sum_{p=0}^{\infty}(-1)^{p} \frac{t^{p} a(p)}{p !}
$$

and then to decide that for noninteger $v$ with $v<p, a(v)$ is given by

$$
a(v)=\int_{0}^{\infty} d t \frac{t^{p-v-1}}{\Gamma(p-v)}(-1)^{p} \frac{d^{p}}{d t^{p}}[J(t)]
$$

It is interesting to notice that this procedure will, in principle, only work if the function $J(t)$ has a finite radius of convergence and has an analytic extension to the real axis. In the case of formulas (114) and (115), $f(t, \lambda) \log f(t, \lambda)$ has a zero radius of convergence (see Appendix A) and so the series expansion (116) does not define a unique function $J(t)$. In the polymer problem the function to use is $f(t, \lambda)$ defined by $(68)$, but there are other functions which have exactly the same series expansion (116) as this function and these would give an incorrect interpolation for the moments.

To learn more about the character of the interpolation, let us consider the low-temperature expansion of (113)-(115). Details of these expansions are given in Appendix A. For $v<0$ the low-temperature behavior can be shown to be (A19)

$$
\begin{aligned}
\left\langle Z_{n+1}^{v}\right\rangle & -\left\langle Z_{n}^{v}\right\rangle^{2} \\
= & \varepsilon \exp \left(v^{2} \frac{2^{n-1}}{T^{2}}\right)\left[-\frac{2^{n-1} v^{2}}{T^{2}}-\frac{1}{2}\right. \\
& \left.-\log \left(\frac{(2 \pi)^{1 / 2} 2^{n / 2}}{T}\right)+\frac{v \Gamma^{\prime}(-v)}{\Gamma(-v)}+\log \Gamma(-v)+\cdots\right]
\end{aligned}
$$

So, the dependence upon $T$ is dominated by the exponential factor.

For $v>0$ one can show that the leading behavior is of the form

$$
\left\langle Z_{n+1}^{v}\right\rangle-\left\langle Z_{n}^{v}\right\rangle^{2}=\varepsilon \exp \left(\frac{2^{n-1} v^{2}}{T^{2}}\right)
$$

So, again the first correction is exponential in termperature. 
We see that the expressions for $v>0$ and $v<0$ are quite different. It is, however, possible to reconcile them by considering that as $T \rightarrow 0$, the ratio $v / T$ remains fixed. Then from (111), by choosing $t=\exp \left(\sqrt{2^{n}} u / T\right)$,

$$
\begin{aligned}
\left\langle Z_{n+1}^{v}\right\rangle & -\left\langle Z_{n}^{v}\right\rangle^{2} \\
= & -\varepsilon v \frac{\sqrt{2^{n}}}{T} \int_{-\infty}^{+\infty} \exp \left(\frac{-\sqrt{2^{n}} u v}{T}\right) d u \\
& \times \int_{u}^{\infty} \frac{\exp \left(-z^{2} / 2\right)}{(2 \pi)^{1 / 2}} d z \log \left(\int_{u}^{\infty} \frac{\exp \left(-z^{2} / 2\right)}{(2 \pi)^{1 / 2}} d z\right)
\end{aligned}
$$

This expression shows that as $v$ and $T$ are small, the result depends only on the ratio $v / T$. For $v / T \rightarrow 0$, one recovers the free energy result (71), whereas for $v / T \rightarrow \pm \infty$ it becomes consistent with (118) and (119). So we see that the limits $v \rightarrow 0$ and $T \rightarrow 0$ do not commute and that it is only if one takes $v / T \rightarrow 0$ as $T \rightarrow 0$ that the replica approach leads to the right free energy.

It is interesting to notice how the width $\Delta_{n}$ of the distribution of $\log Z_{n}$ behaves. Expanding in powers of $v$, it is easy to show that

$$
\begin{aligned}
\log \left\langle Z_{n}^{v}\right\rangle & =v\left\langle\log Z_{n}\right\rangle+\frac{v^{2}}{2}\left[\left\langle\log ^{2} Z_{n}\right\rangle-\left\langle\log Z_{n}\right\rangle^{2}\right]+\cdots \\
& =v\left\langle\log Z_{n}\right\rangle+\frac{v^{2} \Delta_{n}^{2}}{2}+\cdots
\end{aligned}
$$

Hence $\Delta_{n}^{2}$ can be computed by expanding either Eq. (113) or (114) in powers of $v$, to give

$$
\begin{aligned}
\Delta_{n}^{2}= & \frac{L}{T^{2}}+2 L \varepsilon \sum_{i=1}^{n-1} \frac{\Gamma^{\prime}(1)}{2^{i}} \int \frac{d t}{t} f\left(t, \frac{\sqrt{2^{i}}}{T}\right) \log f\left(t, \frac{\sqrt{2^{i}}}{T}\right) \\
& -L \varepsilon \sum_{i=1}^{n-1} \frac{1}{2^{i}} \int \frac{d t}{t}(\log t) f\left(t, \frac{\sqrt{2^{i}}}{T}\right) \log f\left(t, \frac{\sqrt{2^{i}}}{T}\right)
\end{aligned}
$$

where $L=2^{n-1}$ is the length of the polymer.

The first summation in Eq. (122) is proportional to the free energy per unit length of the polymer $F_{n}$ given by (70). Hence, as $n \rightarrow \infty$, this term is proportional to $L$. The second summation does not tend to a finite limit as $n \rightarrow \infty$. Instead, as $n \rightarrow \infty$ (i.e., $\lambda \rightarrow \infty$ ), it can be shown, using the asymptotic expansion of $f(t, \lambda)$ given in Appendix A, (A8), that each term in the series tends to $-2 \varepsilon K_{2}$ [where $K_{2}$ is defined in Eq. (73)]. Hence, this 
last term in Eq. (122) is proportional, for large $n$, to $L \log L$. Indeed, as $n$ increases,

$$
\Delta_{n}^{2} \rightarrow \frac{L}{T^{2}}\left[1-2 T \Gamma^{\prime}(1) F_{n}(T)-2 \varepsilon K_{2}\right]-\frac{2 \varepsilon K_{2}}{T^{2} \log 2} L \log L
$$

We see that as $L$ increases, the corrections increase faster than the leading order and so this expression can only be trusted for $\varepsilon \log L \ll 1$. For $\varepsilon \log L \gg 1$, one should "renormalize" the problem as was done in ref. 23. It is, however, easy to show that (123) is consistent with the results of ref. 23 , which claimed that in the limit $n \rightarrow \infty$, the width $\Delta_{n}$ behaves for large $L$ as $L^{\omega}$ and that $\Delta_{n+1} / \Delta_{n} \rightarrow \lambda_{n}=2^{\omega}$. If we consider from (123) the ratio $\Delta_{n+1} / \Delta_{n}$, we find that

$$
\frac{\Delta_{n+1}}{\Delta_{n}}=2^{1 / 2}\left(1-\varepsilon K_{2}\right)
$$

and

$$
\omega=\frac{1}{2}-\frac{\varepsilon K_{2}}{\log 2}
$$

\section{GENERALIZATION TO OTHER HIERARCHICAL LATTICES}

The results obtained so far have been restricted to the kind of hierarchical lattice described in Section 2 , characterized by the lattice parameter $b$. It is possible to extend these results to other sorts of hierarchical lattice.

An obvious development is to follow the prescription for building the lattice given in Section 2, but to allow more freedom by having $a$ bonds in each branch, rather than fixing this at two. The results obtained will then simply be modified by replacing the characteristic $\sqrt{2^{n}}$ by $\sqrt{a^{n}}$.

The hierarchical lattices discussed so far can only be constructed for integer $b$. The $\varepsilon$ expansion done in Sections 5-7 relies on the analytic continuation of the properties to noninteger values of $b$, although these lattices are not physically realizable. This analytic continuation could produce unphysical results (for example, negative specific heat). We will now see that it is possible to construct physical lattices, which are described by a continuous parameter similar to $b$, to which the results obtained in Sections 5-7 can be extended.

Another way of building a lattice similar to the abstract $b=1+\varepsilon$ lattice is to introduce a probability $p$. Suppose that the $(n+1)$ th generation of the lattice is given by dividing each bond at the $n$th generation into two bonds 


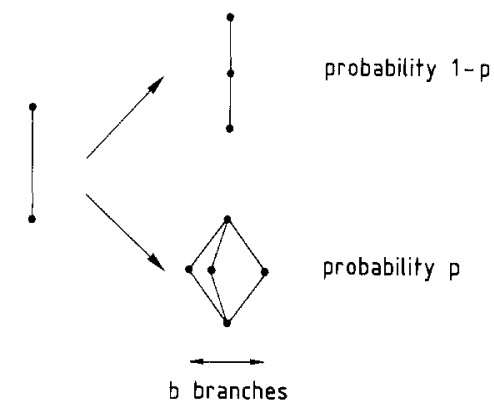

Fig. 6. The iterative construction of the hierarchical lattice characterized by the probability $p$.

with probability $1-p$ and by introducing $b$ branches of two bonds with probability $p$ (see Fig. 6). This lattice can be built for any value of $p$. If we choose $p$ to be small, this lattice is close to the original lattice with $b=1$ and so should be similar to the $b=1+\varepsilon$ lattice, but with $p$ as the small parameter.

For this lattice the recursion corresponding to (5) is

$$
\begin{aligned}
Z_{n+1}= & Z_{n}^{(1)} Z_{n}^{(2)} \quad \text { with probability } 1-p \\
= & Z_{n}^{(1)} Z_{n}^{(2)}+Z_{n}^{(3)} Z_{n}^{(4)}+\cdots+Z_{n}^{(2 b-1)} Z_{n}^{(2 b)} \\
& \quad \text { with probability } p
\end{aligned}
$$

The free energy can be obtained by evaluating $\log \left\langle Z_{n}^{\nu}\right\rangle$ and then taking the limit $v \rightarrow 0$,

$$
F_{n}=-T\left\langle\log Z_{n}\right\rangle=-T \lim _{v \rightarrow 0} \frac{\log \left\langle Z_{n}^{v}\right\rangle}{v}
$$

Let us therefore consider $\left\langle Z_{n+1}^{v}\right\rangle$ with $v<0$. Using recursion (126), we can write this as

$$
\begin{aligned}
\left\langle Z_{n+1}^{v}\right\rangle & =\left\langle Z_{n}^{v}\right\rangle^{2}-p\left\langle\left(Z_{n}^{(1)} Z_{n}^{(2)}\right)^{v}-\left(Z_{n}^{(1)} Z_{n}^{(2)}+\cdots+Z_{n}^{(2 b-1)} Z_{n}^{(2 b)}\right)^{v}\right\rangle \\
& =\left\langle Z_{n}^{v}\right\rangle^{2}-p \int_{0}^{\infty} \frac{d t t^{-v-1}}{\Gamma(-v)}\left[f\left(t, \frac{\sqrt{2^{n}}}{T}\right)-f\left(t, \frac{\sqrt{2^{n}}}{T}\right)^{b}\right]+O\left(p^{2}\right)
\end{aligned}
$$

where we have used the integral relation of Eq. (88a) and noted that when $p=0$ we have the same probability distribution of $Z_{n}$ as with our original lattice with $b=1$. 
Taking the logarithm and then carrying out the recursion formula, with the intial condition that

$$
\left\langle Z_{1}^{v}\right\rangle=e^{v^{2} / 2 T^{2}}
$$

we see that

$$
\begin{aligned}
\frac{\log \left\langle Z_{n}^{v}\right\rangle}{2^{n-1}}= & \frac{v^{2}}{2 T^{2}}-p \sum_{i=1}^{n-1} \frac{1}{2^{i}} \int_{0}^{\infty} d t \\
& \times \frac{t^{-v-1}}{\Gamma(-v)}\left[f\left(t, \frac{\sqrt{2^{i}}}{T}\right)-f\left(t, \frac{\sqrt{2^{i}}}{T}\right)^{b}\right] \exp \left(\frac{-v^{2} 2^{i-1}}{T^{2}}\right)
\end{aligned}
$$

and so finally taking the limit $v \rightarrow 0$, we obtain the average free energy per unit length for this lattice:

$$
F_{n}=\operatorname{Tp} \sum_{i=1}^{n-1} \frac{1}{2^{i}} \int_{0}^{\infty} \frac{d t}{t}\left[f\left(t, \frac{\sqrt{2^{i}}}{T}\right)^{b}-f\left(t, \frac{\sqrt{2^{i}}}{T}\right)\right]
$$

So the formula for the free energy of this lattice is very similar to that for the lattice considered originally $(70)$, the only difference being the replacement of $f\left(t, \sqrt{2^{i}} / T\right) \log f\left(t, \sqrt{2^{i}} / T\right)$ in the original formula by $f\left(t, \sqrt{2^{i}} / T\right)^{6}-f\left(t, \sqrt{2^{i}} / T\right)$. Making this replacement in the other formulas for moments and overlaps will yield correct answers for the new lattice.

Hence we see that the results obtained for the type of hierarchical lattice considered initially can easily be extended to another more physical type of hierarchical lattice.

\section{FINITE-DIMENSIONAL CASE}

For the hierarchical lattice defined in Section 2 we saw that one could obtain the expression for the first integer moments [Eqs. (39)-(42)] at any temperature. From the knowledge of $\langle Z\rangle$ and $\left\langle Z^{2}\right\rangle$, it was possible to show that:

1. For $b>2$, there exists a phase transition at a certain transition temperature $T_{c}(b)$.

2. For $T>T_{c}(b)$, the free energy $F_{n}(T)$ per unit length is given with probability one by

$$
\lim _{n \rightarrow \infty} F_{n}(T)=-T \log b-\frac{1}{2 T}
$$

3. $T_{c}(b)$ must satisfy the following bounds [Eq. (36)]:

$$
[(2 \log b)]^{-1 / 2} \leqslant T_{c}(b) \leqslant[\log (b-1)]^{-1 / 2}
$$


We are going to show in this section that these results can be extended to finite-dimensional lattices.

Consider a directed polymer on a $(d+1)$-dimensional lattice. At each time step $L$ ( $L$ is the length of the polymer) the polymer occupies a site of a regular hypercubic $d$-dimensional lattice. Suppose that at step $L$, the polymer is on site $\mathbf{r}$. Then at step $L+1$, by definition of the model, the polymer is allowed to occupy any of the $2 d$ neighbors of site $\mathbf{r}$. If $Z_{L}(\mathbf{r})$ is the partition function of a polymer of length $L$ which ends at point $r$, one can write the following recursion relation:

$$
Z_{L+1}(\mathbf{r})=\sum_{j=1}^{2 d}\left\{\exp \left(\varepsilon_{L}(\mathbf{r}) / T\right)\right\} Z_{L}\left(\mathbf{r}+\mathbf{e}_{j}\right)
$$

where $-\varepsilon_{L}(\mathbf{r})$ is a random energy on point $(\mathbf{r}, L)$ of the $(d+1)$-dimensional lattice, and the $\mathbf{e}_{j}$ are the $2 d$ unit vectors which connect $\mathbf{r}$ to its $2 d$ neighbors on the $d$-dimensional hypercubic lattice. Notice that, for convenience, we use here site disorder instead of bond disorder (and that we have changed the sign of the energy).

From the recursion (132), we can calculate the behavior for large $L$ of the first two moments of $Z$.

If one averages (132), one gets

$$
\left\langle Z_{L+1}(\mathbf{r})\right\rangle=\left\langle e^{\varepsilon / T}\right\rangle \sum_{j=1}^{2 d}\left\langle Z_{L}\left(\mathbf{r}+\mathbf{e}_{j}\right)\right\rangle
$$

The largest eigenvalue $\lambda$ of the transfer matrix (133) which relates the $\left\langle Z_{L}(\mathbf{r})\right\rangle$ to the $\left\langle Z_{L+1}(\mathbf{r})\right\rangle$ is given by

$$
\lambda_{1}=2 d\left\langle e^{\varepsilon / T}\right\rangle
$$

Therefore in the limit $L \rightarrow \infty$, one expects that

$$
\lim _{L \rightarrow \infty} \frac{\log \left\langle Z_{L}(\mathbf{r})\right\rangle}{L}=\log \lambda_{1}=\log 2 d+\log \left\langle e^{\varepsilon / T}\right\rangle
$$

To calculate the behavior of $\left\langle Z_{L}^{2}(\mathbf{r})\right\rangle$, one has to write a recursion for the following correlation functions $\left\langle Z_{L}(\mathbf{r}) Z_{L}\left(\mathbf{r}^{\prime}\right)\right\rangle$. From (132) one gets

$$
\begin{aligned}
& \left\langle Z_{L+1}(\mathbf{r}) Z_{L+1}\left(\mathbf{r}^{\prime}\right)\right\rangle \\
& =\left\langle e^{\varepsilon / T}\right\rangle^{2} \sum_{i, j}\left\langle Z_{L}\left(\mathbf{r}+\mathbf{e}_{i}\right) Z_{L}\left(\mathbf{r}^{\prime}+\mathbf{e}_{j}\right)\right\rangle \\
& \quad+\left(\left\langle e^{2 \varepsilon / T}\right\rangle-\left\langle e^{\varepsilon / T}\right\rangle^{2}\right) \delta_{r, \mathbf{r}^{\prime}} \sum_{i, j}\left\langle Z_{L}\left(\mathbf{r}+\mathbf{e}_{i}\right) Z_{L}\left(\mathbf{r}+\mathbf{e}_{j}\right)\right\rangle
\end{aligned}
$$


To find the largest eigenvalue $\lambda_{2}$ of this transfer matrix, we can use the fact that the corresponding eigenvector has the form

$$
\left\langle Z(\mathbf{r}) Z\left(\mathbf{r}^{\prime}\right)\right\rangle=\varphi\left(\mathbf{r}-\mathbf{r}^{\prime}\right)
$$

Therefore the equation (136) gives $\varphi$ and $\lambda$ as

$$
\lambda \varphi(\mathbf{r})=\left\langle e^{\varepsilon / T}\right\rangle^{2} \sum_{j, k} \varphi\left(\mathbf{r}+\mathbf{e}_{j}-\mathbf{e}_{k}\right)+B \delta(\mathbf{r})
$$

where

$$
B=\left[\left\langle e^{2 \varepsilon / T}\right\rangle-\left\langle e^{\varepsilon / T}\right\rangle^{2}\right] \sum_{j, k} \varphi\left(\mathbf{e}_{j}-\mathbf{e}_{k}\right)
$$

The solution $\varphi$ of (138) for arbitrary $B$ can easily be obtained by going to Fourier space,

$$
\varphi(\mathbf{r})=\int \frac{d \mathbf{q}}{(2 \pi)^{d}}[\exp (i \mathbf{q} \cdot \mathbf{r})] G(\mathbf{q})
$$

and one finds

$$
\begin{aligned}
G(\mathbf{q}) & =B\left\{\lambda-\left\langle e^{\varepsilon / T}\right\rangle^{2} \sum_{j, k} \exp \left[i \mathbf{q}\left(\mathbf{e}_{j}-\mathbf{e}_{k}\right)\right]\right\}^{-1} \\
& =B\left[\lambda-\left\langle e^{\varepsilon / T}\right\rangle^{2}\left(\sum_{\mu=1}^{d} 2 \cos q_{\mu}\right)^{2}\right]^{-1}
\end{aligned}
$$

For $\lambda$ to be an eigenvalue and $\varphi$ to be the corresponding eigenvector (138) one needs $B$ to satisfy (139). One has just to replace $\varphi$ given by (140) and (141) to get an equation for the eigenvalues $\lambda$,

$$
\begin{aligned}
1= & {\left[\left\langle e^{2 \varepsilon / T}\right\rangle-\left\langle e^{\varepsilon / T}\right\rangle^{2}\right] \int_{0}^{2 \pi} \frac{d q_{1}}{2 \pi} \cdots \int_{0}^{2 \pi} \frac{d q_{d}}{2 \pi} } \\
& \times\left(\sum_{\mu=1}^{d} 2 \cos q_{\mu}\right)^{2}\left[\lambda-\left\langle e^{\varepsilon / T}\right\rangle^{2}\left(\sum_{\mu=1}^{d} 2 \cos q_{\mu}\right)^{2}\right]^{-1}
\end{aligned}
$$

The largest value eigenvalue $\lambda_{2}$ of (136) is the largest solution of this equation.

Clearly one has for $L \rightarrow \infty$,

$$
\lim _{L \rightarrow \infty} \frac{\log \left\langle Z_{L}^{2}(\mathbf{r})\right\rangle}{L}=\log \lambda_{2}
$$


For $d \leqslant 2$, the largest eigenvalue $\lambda_{2}$ given by (142) is a smooth function of temperature which is always strictly larger than $\left(2 d\left\langle e^{\varepsilon / T}\right\rangle\right)^{2}$ and therefore

$$
\lim _{L \rightarrow \infty} \frac{\log \left\langle Z_{L}^{2}(\mathbf{r})\right\rangle}{L}>2 \lim _{L \rightarrow \infty} \frac{\log \left\langle Z_{L}(\mathbf{r})\right\rangle}{L}
$$

For $d>2$, there exists a critical temperature $T_{2}(d)$ which is given by the condition that

$$
\frac{\left\langle e^{\varepsilon / T_{2}}\right\rangle^{2}}{\left\langle e^{2 \varepsilon / T_{2}}\right\rangle-\left\langle e^{\varepsilon / T_{2}}\right\rangle^{2}}=I_{d}
$$

where

$$
I_{d}=\int_{0}^{2 \pi} \frac{d q_{1}}{2 \pi} \cdots \int_{0}^{2 \pi} \frac{d q_{d}}{2 \pi} \frac{\left(\sum_{\mu=1}^{d} \cos q_{\mu}\right)^{2}}{d^{2}-\left(\sum_{\mu=1}^{d} \cos q_{\mu}\right)^{2}}
$$

and one can show that

$$
\begin{array}{lll}
\text { for } & T>T_{2} & \lambda_{2}=(2 d)^{2}\left\langle e^{\varepsilon / T}\right\rangle^{2}=\lambda_{1}^{2} \\
\text { for } & T<T_{2} & \lambda_{2}>(2 d)^{2}\left\langle e^{\varepsilon / T}\right\rangle^{2}=\lambda_{1}^{2}
\end{array}
$$

So we see that in dimension $d>2$, there is a high-temperature phase where

$$
\lim _{L \rightarrow \infty} \frac{\log \left\langle Z_{L}^{2}\right)}{L}=2 \lim _{L \rightarrow \infty} \frac{\log \left\langle Z_{L}\right\rangle}{L}
$$

This situation is similar to what occurred in the hierarchical lattice (above $T_{2}$, we had $\left\langle Z^{2}\right\rangle /\langle Z\rangle^{2} \rightarrow 1$ as the number $n$, and therefore the length $L$, increases). Here the condition (148) is weaker, since it does not allow us to conclude that the distribution of $Z$ becomes a delta peak in the limit $L \rightarrow \infty$. It is, however, possible to prove (see Appendix B) that for $T>T_{2}$,

$$
\lim _{L \rightarrow \infty} \frac{\log Z_{L}}{L}=\log 2 d+\log \left\langle e^{\varepsilon / T}\right\rangle
$$

with probability 1 . The idea we use in the Appendix to prove (149) is an adaptation to the model studied here of the proof given by Imbrie and Spencer. ${ }^{(27,28)}$ This implies that for $T>T_{2}$, the free energy $F(T)$ per unit length is given with probability 1 by

$$
F(T)=-T \log 2 d-T \log \left\langle e^{\varepsilon / T}\right\rangle
$$


However, it is not known down to what temperature the free energy is given by expression (150). So, as for the hierarchical lattice, one can only deduce from (150) bounds for the transition temperature $T_{c}(d)$ with $b$ replaced by $2 d$ and one gets

$$
(2 \log 2 d)^{-1 / 2}<T_{c}(d)<T_{2}(d)
$$

where the lower bound is obtained again as the temperature where (150) would give a negative entropy and $T_{2}$ is the solution of (145), which for a Gaussian $\rho(\varepsilon)$ becomes

$$
T_{2}(d)=\left[\log \left(1+\frac{1}{I_{d}}\right)\right]^{-1 / 2}
$$

The integral (146) can be related easily to a well-known integral in the theory of random walks ${ }^{(37)}$

$$
I_{d}=-1+d \int_{0}^{2 \pi} \frac{d q_{1}}{2 \pi} \cdots \int_{0}^{2 \pi} \frac{d q_{d}}{2 \pi}\left(d-\sum_{\mu=1}^{d} \cos q_{\mu}\right)^{-1}
$$

and this gives

$$
\begin{aligned}
I_{d} & =\infty & & \text { if } \quad d \leqslant 2 \\
& =0.5164 & & \text { if } \quad d=3 \\
& =\frac{1}{2 d}+\cdots & & \text { for large } d
\end{aligned}
$$

which leads to the following bounds for the transition temperature $T_{c}$ :

$$
\begin{aligned}
& 0.6 \leqslant T_{c} \quad \text { for } d=2 \\
& 0.528 \leqslant T_{c} \leqslant 0.963 \text { for } d=3 \\
& (2 \log 2 d)^{-1 / 2} \leqslant T_{c} \leqslant(\log 2 d)^{-1 / 2} \quad \text { for large } d
\end{aligned}
$$

It is interesting to notice that one can obtain bounds very similar to (155) for the problem of a polymer on a tree with coordination number $z+1$,

$$
(2 \log z)^{-1 / 2} \leqslant T_{c} \leqslant(\log z)^{-1 / 2}
$$

and that in that case the exact solution ${ }^{(1)}$ gives that $T_{c}$ is identical to its lower bound. 


\section{CONCLUSION}

In the present work, we have shown that the problem of a polymer on a random hierarchical lattice can be reduced to the study of stable distributions when one combines random variables in a nonlinear way [see (3) and (5)]. The temperature $T$ enters only in the initial distribution $\pi_{1}(Z)$. In the high-temperature phase the distribution of $\log Z$ becomes a delta function as $n$ increases, whereas in the low-temperature phase the width of this distribution increases with $n$ and $\left\langle\log ^{2} Z\right\rangle-\langle\log Z\rangle^{2} \sim L^{2 \omega}$, where $\omega$ depends on the lattice. ${ }^{(8)}$

We have also seen that for $b=1+\varepsilon$, with $\varepsilon$ small, one can extend to finite temperature the calculation of ref. 23 and calculate thermal properties (free energy, specific heat), (70) and (71), as well as geometrical properties (overlaps), (96), (98), and (100). This expansion method is nevertheless not sufficient to study the phase transition, since the high-temperature phase seems to exist only for $b>2$. However, it allows one to describe the lowtemperature behavior of several quantities: linear specific heat at $T=0$, Eq. (71); anomalous fluctuations of the free energy, Eq. (125) ${ }^{\{23)}$; and a distribution $P_{2}(q)$ of overlaps which consists of a single delta function, Eq. (109).

We have shown that from the knowledge of the first two moments $\langle Z\rangle$ and $\left\langle Z^{2}\right\rangle$ of the partition function, one can give bounds $(36)$ on the transition temperature $T_{c}(b)$ and one can find the exact expression of the free energy above a certain temperature $T_{2}(b)$ [where $\left.T_{2}(b) \geqslant T_{c}(b)\right]$. The argument which leads to the bounds on $T_{c}(b)$ and to the expression of $\langle\log Z\rangle$ in the high-temperature phase can be extended to finite-dimensional lattices (Section 9).

Lastly, in calculating the moments $\left\langle Z^{v}\right\rangle$ of the partition function we obtained an interpolation formula (116)-(117) which allows one to obtain $\left\langle Z^{v}\right\rangle$ for noninteger $y$ from the knowledge of the integer moments $\left\langle Z^{n}\right\rangle$. It would be interesting to study the validity and the limitations of this expression and to compare it which the replica techniques.

There are several questions on this problem of polymers on a hierarchical lattice that we were not able to answer: first, we could not calculate the transition temperature $T_{c}(b)$ for $b>2$. So we have the expression of the free energy in the high-temperature phase, but we do not know down to what temperature it is valid. It would be interesting to find a way of calculating $T_{c}(b)$ for the hierarchical lattice because this could probably be generalized to finite-dimensional lattices.

One of our motivations in studying the problem of polymers on random lattices is that in the mean-field limit, the problem has many properties similar to those of mean-field spin glasses. ${ }^{(29,30)}$ So we wanted to 
know whether properties such as a broad distribution of overlaps or nonself-averaging would persist in lower dimensional systems (for $b$ finite). Our $\varepsilon$ expansion ( $b$ close to 1 ) does not indicate this mean-field property, but we cannot exclude that above a certain value of $b$, these effects appear. It would be interesting to investigate this question because the problem of polymers on random lattices is in many respects a simpler problem than that of spin glasses.

\section{APPENDIX A}

This appendix discusses some of the properties of the function

$$
f(t, \lambda)=\int_{-\infty}^{+\infty} \frac{d u}{(2 \pi)^{1 / 2}} \exp \left(-\frac{1}{2} u^{2}-t e^{\lambda u}\right)
$$

It is organized as follows. First the radius of convergence of the series expansion of $f(t, \lambda)$ about $t=0$ is considered. Next the various asymptotic expansions of $f(t, \lambda)$ for large $\lambda$ are derived. Lastly the method of obtaining the low-temperature expansions used in the main body of the paper is presented.

\section{A1. Radius of Convergence}

It is stated in Section 7 that the series expansion of $f(t, \lambda)$ about $t=0$ has a zero radius of convergence. This can easily be verified by considering the form of the Taylor series about $t=0$ :

$$
f(t, \lambda)=\sum_{k=0}^{\infty} \frac{t^{k}}{k !} f^{(k)}(0, \lambda)
$$

The derivatives of $f(t, \lambda)$ evaluated at $t=0$ are

$$
f^{(k)}(0, \hat{\lambda})=\left.(-1)^{k} \int \exp \left(k \lambda u-\frac{1}{2} u^{2}-t e^{\hat{\lambda} u}\right) \frac{d u}{(2 \pi)^{1 / 2}}\right|_{t=0}=(-1)^{k} e^{k^{2} \hat{\lambda}^{2} / 2}
$$

Hence the coefficients of the series expansion about $t=0$ increase with $k$ faster than any exponential, and so the series (A2) has a zero radius of convergence. The fact that the derivatives of $f(t, \lambda)$ with respect to $t$, evaluated at $t=0$, have such a simple form allows the integer moments (115) and overlaps $Q(2+p),(97),(102)$, and (103), to be calculated relatively easily. 


\section{A2. Asymptotic Expansion}

We now proceed to consider the asymptotic expansion (large $\lambda$, which corresponds to the low-temperature limit) of the function $f(t, \lambda)$. The asymptotic form of $f(t, \lambda)$ depends upon the range of $t$ considered. It is necessary to consider three cases: (i) $\log t \sim \lambda$, (ii) $\log t \sim \lambda^{2}$ with $\log t>0$, and (iii) $-p \lambda^{2}<\log t<-(p-1) \lambda^{2}$ with $p$ a positive integer. These three cases will now be considered separately and the relevant asymptotic form of $f(t, \lambda)$ obtained.

(i) $\log t \sim \lambda$

Consider making the change of variable $t=e^{\lambda z}$. If now the derivative of $f(z, \lambda)$ with respect to $\lambda$ is considered, it is found to have the form

$$
\frac{d f}{d \lambda}(z, \lambda)=-\int_{-\infty}^{\infty} \frac{d u}{(2 \pi)^{1 / 2}}(u+z) \exp \left\{\lambda(u+z)-\frac{1}{2} u^{2}-\exp [\lambda(u+z)]\right\}
$$

and so only the immediate region of $u=-z$ contributes substantially to the integral. So, letting $u=-z+v / \lambda$, (A4) becomes

$$
\frac{d f}{d \lambda}(z, \lambda)=-\frac{\exp \left(-z^{2} / 2\right)}{\lambda^{2}} \int \frac{d v}{(2 \pi)^{1 / 2}} v \exp \left[v+\frac{z v}{\lambda}-\frac{v^{2}}{2 \lambda^{2}}-\exp (v)\right]
$$

Expanding the bracketed exponentials in powers of $v / \lambda$ and noting that

$$
\int_{-\infty}^{\infty} v^{p} \exp [v-\exp (v)] d v=\Gamma^{(p)}(1)
$$

[where $\Gamma^{(k)}(1)$ is the $k$ th derivative of the gamma function evaluated at 1 ], one finds

$$
\frac{d f}{d \lambda}(z, \lambda)=-\frac{e^{-z^{2} / 2}}{(2 \pi)^{1 / 2} \lambda^{2}}\left[\Gamma^{(1)}(1)+\frac{z}{\lambda} \Gamma^{(2)}(1)+\frac{1}{2 \lambda^{2}}\left(z^{2}-1\right) \Gamma^{(3)}(1)+O\left(\frac{1}{\lambda^{3}}\right)\right]
$$

Hence we see that $f(t, \lambda)$ can be written as

$$
\begin{aligned}
f(t, \lambda)= & \int_{\log t / \lambda}^{\infty} \frac{d u}{(2 \pi)^{1 / 2}}\left(\exp \frac{-u^{2}}{2}\right)+\frac{\exp \left(-\log ^{2} t / 2 \lambda^{2}\right)}{(2 \pi)^{1 / 2}} \\
& \times\left[\frac{\Gamma^{(1)}(1)}{\lambda}+\frac{\Gamma^{(2)}(1)}{2 \lambda^{2}} \frac{\log t}{\lambda}+\frac{\Gamma^{(3)}(1)}{6 \lambda^{3}}\left(\frac{\log ^{2} t}{\lambda^{2}}-1\right)+\cdots\right]
\end{aligned}
$$


where it has been noted that

$$
f(z, \infty)=\int_{z}^{\infty} \frac{d u}{(2 \pi)^{1 / 2}} e^{-u^{2} / 2}
$$

It will be found useful to define

$$
F(z)=\int_{z}^{\infty} \frac{d u}{(2 \pi)^{1 / 2}} e^{-u^{2} / 2}
$$

(ii) $\log t \sim \lambda^{2}$ with $\log t>0$

To derive the asymptotic form of $f(t, \lambda)$ in this case, consider the change of variable $z=t e^{i u}$. This converts (A1) to

$$
f(t, \lambda)=\frac{1}{(2 \pi)^{1 / 2} \lambda}\left(\exp \frac{-\log ^{2} t}{2 \lambda^{2}}\right) \int_{0}^{\infty} d z z^{(\log t) / \lambda^{2}-1} \exp \left(-z-\frac{\log ^{2} z}{2 \lambda^{2}}\right)
$$

The integrand is exponentially damped for large $z$. So it is valid to expand the final exponential for small arguments to obtain

$$
\begin{aligned}
f(t, \hat{\lambda})= & \frac{1}{(2 \pi)^{1 / 2} \lambda}\left(\exp \frac{-\log ^{2} t}{2 \lambda^{2}}\right) \\
& \times \int_{0}^{\infty} d z z^{(\log t) / \lambda^{2}-1}\left[1-\frac{\log ^{2} z}{2 \lambda^{2}}+O\left(\frac{1}{\lambda^{4}}\right)\right] \exp (-z) \\
= & \frac{1}{(2 \pi)^{1 / 2} \lambda}\left(\exp \frac{-\log ^{2} t}{2 \lambda^{2}}\right) \\
& \times\left[\Gamma\left(\frac{\log t}{\lambda^{2}}\right)-\frac{1}{2 \lambda^{2}} \Gamma^{(2)}\left(\frac{\log t}{\lambda^{2}}\right)+O\left(\frac{1}{\lambda^{4}}\right)\right]
\end{aligned}
$$

(iii) $-p \lambda^{2}<\log t<-(p-1) \lambda^{2}, p$ a positive integer

It has been shown for a very similar integral (Appendix of ref. 32 ) that, for this range of $t$, the asymptotic form of $f(t, \lambda)$ is

$$
\begin{aligned}
f(t, \lambda)= & 1-t e^{\lambda^{2} / 2}+\frac{t^{2}}{2 !} e^{2 \lambda^{2}}+\cdots+(-1)^{p-1} \frac{t^{p-1}}{(p-1) !} e^{(p-1)^{2} \lambda^{2} / 2} \\
& +\frac{1}{(2 \pi)^{1 / 2} \lambda} e^{-\log ^{2} t / 2 \lambda^{2}}\left[\Gamma\left(\frac{\log t}{\lambda^{2}}\right)-\frac{1}{2 \lambda^{2}} \Gamma^{(2)}\left(\frac{\log t}{\lambda^{2}}\right)+O\left(\frac{1}{\lambda^{4}}\right)\right]
\end{aligned}
$$




\section{A3. Low-Temperature Expansions}

Having obtained the asymptotic forms of $f(t, \lambda)$, we will now consider how to obtain the low-temperature expansions stated in Eqs. (71), (99), (100), and (118)-(120). In each case it is necessary to calculate an integral over the variable $t$ with an integrand which is a product of some power of $t$ and a simple function of $f(t, \lambda)$. We have just seen that $f(t, \lambda)$ has different asymptotic forms depending upon the range of $t$. Hence, to evaluate the required integrals we must first determine which of the asymptotic forms of $f(t, \lambda)$ it is necessary to use. We will see that for all the integrals required, the integrand is sharply peaked in one of the three regions of $t$ considered. Hence it is only necessary to use one of the three possible asymptotic forms of $f(t, \lambda)$ for the calculation of the integrals. So, the lowtemperature expansions fall into three classes, depending upon which range of $t$ dominates the integrand. We will deal with each class of expansion separately.

(i) In the low-temperature expansions of free energy [Eq. (71)], $\left\langle q_{2}\right\rangle$ [Eq. (99)], $\left\langle q_{m}\right\rangle$ [Eq. (100)], and moment with $v=0$ [Eq. (120)], the integrals are dominated by $t$ in the range $\log t \sim \lambda$. Consider the calculation of the free energy as an example of this class, in which we need to consider the integral

$$
\int_{0}^{\infty} \frac{d t}{t} f(t, \lambda) \log f(t, \lambda)=\int_{-\infty}^{\infty} d(\log t) f(t, \lambda) \log f(t, \lambda)
$$

Consider first case (i), i.e., $\log t \sim \lambda$. Then, setting $\log t=\lambda z$, the integrand behaves as

$$
f(t, \lambda) \log f(t, \lambda) \sim F(z) \log F(z)
$$

where $F(z)$ is defined in (A10).

Now take case (ii), $\log t \sim \lambda^{2}$, where the integrand has the behavior

$$
f(t, \lambda) \log f(t, \lambda) \sim \frac{1}{\lambda} e^{-\log ^{2} t / 2 \lambda^{2}}\left(\frac{\log ^{2} t}{2 \lambda^{2}}+\log \lambda\right)
$$

This has its maximum value near $(\log t) / \lambda^{2}=0$, which is the boundary of region (ii), and that means that the range of $t$ which dominates the integral is elsewhere.

Finally let us consider case (iii). For $-p \lambda^{2}<\log t<-(p-1) \lambda^{2}$,

$$
\begin{aligned}
f(t, \lambda) & \log f(t, \lambda) & \\
& \simeq-t e^{\lambda^{2} / 2} & \text { for } p>1 \\
& \simeq \frac{1}{(2 \pi)^{1 / 2} e^{-\log ^{2} t / 2 \lambda^{2}} \Gamma\left(\frac{\log t}{\lambda^{2}}\right)+\cdots} & \text { for } \quad p=1
\end{aligned}
$$


Each of these terms is exponentially small, i.e., $\sim \exp \left(-\right.$ const $\left.\cdot \lambda^{2}\right)$, and so again the integrand is very much smaller for this range of $t$ than for case (i). Hence we see that the integral (A14) is dominated by $\log t \sim \lambda$. Similar consideration shows that this is also true for the integrals in (99) and (120).

Now it just remains to evaluate (A14) using the appropriate asymptotic form of $f(t, \lambda)$, (A8):

$$
\begin{aligned}
& \int_{0}^{\infty} \frac{d t}{t} f(t, \lambda) \log f(t, \lambda) \\
&=\lambda \int_{-\infty}^{\infty} d z\left\{F(z) \log F(z)+\frac{1}{\lambda} \frac{e^{-z^{2} / 2}}{(2 \pi)^{1 / 2}} \Gamma^{(1)}(1)[1+\log F(z)]\right. \\
&+\frac{1}{2 \lambda^{2}}\left[\frac{z e^{-z^{2} / 2}}{(2 \pi)^{1 / 2}} \Gamma^{(2)}(1)[1+\log F(z)]+\frac{e^{-z^{2}}}{2 \pi} \frac{\Gamma^{(1)^{2}}(1)}{F(z)}\right] \\
&+\frac{1}{\lambda^{3}}\left[\frac{z^{2}-1}{2} \frac{e^{-z^{2} / 2}}{(2 \pi)^{1 / 2}} \frac{\Gamma^{(3)}(1)}{3}[1+\log F(z)]\right. \\
&\left.\left.+\frac{z e^{-z^{2}}}{4 \pi} \frac{\Gamma^{(1)}(1) \Gamma^{(2)}(1)}{F(z)}-\frac{e^{-3 z^{2} / 2}}{(2 \pi)^{3 / 2}} \frac{\Gamma^{(1)^{3}}(1)}{6 F^{2}(z)}\right]\right\}
\end{aligned}
$$

Hence, via (70), we obtain

$$
\begin{aligned}
F_{n}= & \varepsilon\left[-K_{1} \sum_{i=1}^{n-1} \frac{1}{\sqrt{2^{i}}}+\left(\frac{\Gamma^{(1)^{2}}(1)-\Gamma^{(2)}(1)}{2}\right) K_{1} T^{2} \sum_{i=1}^{n-1} \frac{1}{2^{3 i / 2}}\right. \\
& +\left\{-\frac{\Gamma^{(3)}(1)}{3}+\Gamma^{(1)}(1) \Gamma^{(2)}(1)-\frac{2 \Gamma^{(1)^{3}}(1)}{3}\right\} K_{2} T^{3} \sum_{i=1}^{n-1} \frac{1}{4^{i}}+O\left(T^{4)}\right]
\end{aligned}
$$

where $K_{1}$ and $K_{2}$ are as given in (72), (73).

The low-temperature expansions of $\left\langle q_{2}\right\rangle,\left\langle q_{m}\right\rangle$, and the moments with $v=0$ are obtained in exactly the same manner.

(ii) The integrals contained in the expression for the negative moments of the partition function and the overlaps $Q(2+v)$ with $v<-2$ are dominated in the low-temperature limit by $t$ in the range $\log t \sim+\lambda^{2}$. As an example, we will consider the negative moments (113), (118), for which we must evaluate the integral

$$
\begin{aligned}
& \int_{0}^{\infty} d t \frac{t^{-v-1}}{\Gamma(-v)} f(t, \lambda) \log f(t, \lambda) \\
& \quad=\int_{-\infty}^{\infty} \frac{d(\log t)}{\Gamma(-v)} t^{-v} f(t, \lambda) \log f(t, \lambda)
\end{aligned}
$$


First we show that the integrand is sharply peaked for $\log t \sim+\lambda^{2}$ and then we will derive (118).

For $\log t \sim \lambda$ we again make the change of variable $\log t=\lambda z$ to see that the integrand behaves as

$$
t^{-v} f(t, \lambda) \log f(t, \lambda) \sim e^{-v \lambda z} F(z) \log F(z)
$$

For case (ii), with $\log t \sim+\lambda^{2}$ the integrand has the form

$$
t^{-v} f(t, \lambda) \log f(t, \lambda) \sim \frac{e^{-v \log t}}{\lambda} e^{-\log ^{2} t / 2 \lambda^{2}}\left(\frac{\log ^{2} t}{2 \lambda^{2}}+\log \lambda\right)
$$

This has a maximum near $\log t=-v \lambda^{2}$ and at this point it takes a value of the order $\lambda \exp \left(v^{2} \lambda^{2} / 2\right)$.

Lastly, for case (iii), $-p \lambda^{2}<\log t<-(p-1) \lambda^{2}$,

$$
\begin{array}{rlrl}
t^{-v} f(t, \lambda) \log f(t, \lambda) & & \\
& \sim-e^{-v \log t} t e^{\lambda^{2} / 2} & \text { for } p>1 \\
& \sim e^{-v \log t} \frac{1}{(2 \pi)^{1 / 2} \lambda} e^{-\log ^{2} t / 2 \lambda^{2}} \Gamma\left(\frac{\log t}{\lambda^{2}}\right) & & \text { for } p=1
\end{array}
$$

Again all these terms are exponentially small, $\sim \exp \left(-\right.$ const $\left.\cdot \lambda^{2}\right)$. Hence the integrand is seen to be peaked for $\log t \sim \lambda^{2}$.

Now we can evaluate (A17) using the asymptotic form of $f(t, \lambda)$ for case (ii), (A12). Using the change of variable $t=\exp \left(\lambda^{2} z\right)$, we obtain

$$
\begin{aligned}
\int_{0}^{\infty} \frac{d t t^{-v-1}}{\Gamma(-v)} f(t, \lambda) \log f(t, \lambda) & \\
= & \frac{\lambda}{\Gamma(-v)} \frac{1}{(2 \pi)^{1 / 2}} \int_{-\infty}^{\infty} d z e^{-v \lambda^{2} z} e^{-\lambda^{2} z^{2} / 2}\left[-\Gamma(z) \log \left[(2 \pi)^{1 / 2} \lambda\right]\right. \\
& \left.\quad-\frac{\lambda^{2} z^{2}}{2} \Gamma(z)+\Gamma(z) \log \Gamma(z)+\frac{z^{2}}{4} \Gamma^{(2)}(z)+\cdots\right]
\end{aligned}
$$

This can be simplified by making the change of variable $y=z+v$ and then expanding the gamma functions for small $y$, the exponential damping out any contribution from large $y$. Then performing the remaining integrals, one obtains, using (111),

$$
\begin{aligned}
\left\langle Z_{n+1}^{v}\right\rangle & -\left\langle Z_{n}^{v}\right\rangle^{2} \\
= & \varepsilon \exp \left(\frac{v^{2} 2^{n-1}}{T^{2}}\right)\left\{-\frac{2^{n-1} v^{2}}{T^{2}}-\log \left(\frac{(2 \pi)^{1 / 2} 2^{n / 2}}{T}\right)\right. \\
& \left.+\frac{v \Gamma^{\prime}(-v)}{\Gamma(-v)}+\log \Gamma(-v)-\frac{1}{2}\right\}
\end{aligned}
$$


The low-temperature expansion of the overlaps $Q(2+v)$ with $v<-2$ is obtained in the same manner.

(iii) Lastly, in the low-temperature expansions of the positive moments (119) and the overlaps $Q_{n}(2+v)$ for $v>-2$, the integrals are dominated by $t$ in the range $-(p-1) \hat{\lambda}^{2}>\log t>-p \hat{\lambda}^{2}$. As an example, we consider the low-temperature expansion of the positive moments with $m>v>m-1$. Here we need to evaluate the integral

$$
\begin{aligned}
& \int_{0}^{\infty} d t t^{m-v-1}(-1)^{m} \frac{d^{m}}{d t^{m}}[f(t, \lambda) \log f(t, \lambda)] \\
& \quad=\int_{-\infty}^{\infty} d(\log t) e^{(m-v) \log t}(-1)^{m} \frac{d^{m}}{d t^{m}}[f(t, \lambda) \log f(t, \lambda)]
\end{aligned}
$$

For $\log t \sim \lambda$, with $t=e^{\lambda z}$, the integrand behaves for large $\lambda$ as

$$
t^{m-v} \frac{d^{m}}{d t^{m}}[f(t, \lambda) \log f(t, \lambda)] \sim e^{-v \log t}
$$

Second, we consider $\log t \sim \lambda^{2}$; then the integrand behaves as

$$
t^{m-v} \frac{d^{m}}{d t^{m}}[f(t, \lambda) \log f(t, \lambda)] \sim e^{-v \log t} e^{-\log ^{2} t / 2 \lambda^{2}}
$$

which is of the form $\exp \left(-\right.$ const $\left.\cdot \lambda^{2}\right)$.

Lastly we look at $-p \hat{\lambda}^{2}<\log t<-(p-1) \hat{\lambda}^{2}$. In this range of $t$ the integrand has the form

$$
t^{m-\nu} \frac{d^{m}}{d t^{m}}[f(t, \lambda) \log f(t, \lambda)] \sim e^{-v \log t} e^{-\log ^{2} t / 2 \lambda^{2}}
$$

This has a maximum when $-\lambda^{2} v=\log t$, when it takes the value $\exp \left(v^{2} \lambda^{2} / 2\right)$. This is much greater than the value of the integrand in either of the other regions. So we see that the correct form of $f(t, \lambda)$ to use is that for $t$ in the range $-m \lambda^{2}<\log t<-(m-1) \lambda^{2}$. Having determined the dominant range of $t$, it is now straightforward to evaluate the lowtemperature expansion for the positive moments from Eq. (114), using the same approach to evaluating the integrals as explained for the treatment of (A18). This yields the results of Eq. (119). The overlaps $Q_{n}(2+v)$ for $v>-2$ can be evaluated by the same method.

\section{APPENDIX B}

In this appendix we will show that for the model defined by (132)

$$
Z_{L+1}(\mathbf{r})=\sum_{j=1}^{2 d}\left\{\exp \left[\varepsilon_{L}(\mathbf{r}) / T\right]\right\} Z_{L}\left(\mathbf{r}+\mathbf{e}_{j}\right)
$$


with $Z_{0}(\mathbf{r})=1$, one has, for $T>T_{2}\left[T_{2}\right.$ is given by (145)]

$$
\lim _{L \rightarrow \infty} \frac{\log Z_{L}}{L}=\lim _{L \rightarrow \infty} \frac{\log \left\langle Z_{L}\right\rangle}{L}
$$

with probability 1 .

We will first show that (B2) is true with a nonzero probability [see (B8)]. Then we will explain how this nonzero probability can be made as close to 1 as wanted.

First let us calculate, for $T>T_{2}$, the ratio $\left\langle Z_{L}^{2}\right\rangle\left\langle\left\langle Z_{L}\right\rangle^{2}\right.$. Following the idea of ref. 27 , one can write

$$
\left\langle Z_{L}^{2}\right\rangle /\left\langle Z_{L}\right\rangle^{2}=\left[\sum_{W_{1}} \sum_{W_{2}}\left(\left\langle e^{2 \varepsilon / T}\right\rangle /\left\langle e^{\varepsilon / T}\right\rangle^{2}\right)^{N\left(W_{1}, W_{2}\right)}\right] / \sum_{W_{1}} \sum_{W_{2}} 1
$$

where $W_{1}$ and $W_{2}$ are two random walks of length $L$ on a hypercubic lattice of dimension $d$ starting at $O$ and $N\left(W_{1}, W_{2}\right)$ is the number of intersections of the two walks (defined as the number of time steps at which the two walkers visit the same site).

In dimension $d>2$ and for $L \rightarrow \infty$, one can calculate the probability $P_{m}$ that two random walks meet exactly $m$ times. One finds, using the techniques explained in ref. 37 , that

$$
\begin{aligned}
P_{m} & =A^{m-1}(1-A) \\
P_{0} & =0
\end{aligned}
$$

where the constant $A$ is given by

$$
\begin{aligned}
A & =1-\left[\frac{1}{(2 \pi)^{d}} \iint \frac{d q_{1} \cdots d q_{d}}{1-\left(1 / d^{2}\right)\left(\sum_{\mu=1}^{d} \cos q_{\mu}\right)^{2}}\right]^{-1} \\
& =\frac{I_{d}}{I_{d}+1}
\end{aligned}
$$

where $I_{d}$ is defined in (146). Notice that $P_{0}=0$ because the two walks meet at least once, since they meet at their starting point. Then from (B3) and (B4), one gets that

$$
\lim _{L \rightarrow \infty} \frac{\left\langle Z_{L}^{2}\right\rangle}{\left\langle Z_{L}\right\rangle^{2}}=B
$$

where $B$ is given by

$$
B=\sum_{m=0}^{\infty} P_{m}\left(\frac{\left\langle e^{2 \varepsilon / T}\right\rangle}{\left\langle e^{\varepsilon / T}\right\rangle^{2}}\right)^{m}=\frac{(1-A)\left\langle e^{2 \varepsilon / T}\right\rangle /\left\langle e^{\varepsilon / T}\right\rangle^{2}}{1-A\left\langle e^{2 \varepsilon / T}\right\rangle /\left\langle e^{\varepsilon / T}\right\rangle^{2}}
$$


From the definition of $T_{2}$ [Eqs. (145) and (146)], one can see that when $T$ varies from $\infty$ to $T_{2}, B$ goes from 1 to $\infty$.

Let us now show that (B6) implies that

$$
\lim _{L \rightarrow \infty}\left(\frac{\log Z_{L}}{L}\right)=\lim _{L \rightarrow \infty} \frac{\log \left\langle Z_{L}\right\rangle}{L} \text { with probability at least } \frac{1}{B}
$$

To do that, we will use ${ }^{(27)}$ Chebyschev's inequality: from (B6), it is clear that for any constant $C$

$$
\lim _{L \rightarrow \infty}\left\langle\left(\frac{Z_{L}}{\left\langle Z_{L}\right\rangle}-C\right)^{2}\right\rangle=B-2 C+C^{2}
$$

Therefore $^{(27)}$

$$
\operatorname{Prob}\left[\left|\frac{Z_{L}}{\left\langle Z_{L}\right\rangle}-C\right|<D\right] \geqslant 1-\left(\frac{B-2 C+C^{2}}{D^{2}}\right)
$$

As long as $D<C$, the fact that $\left|C-Z_{L} /\left\langle Z_{L}\right\rangle\right|<D$ implies that

$$
\lim _{L \rightarrow \infty} \frac{\log Z_{L}}{L}=\lim _{L \rightarrow \infty} \frac{\log \left\langle Z_{L}\right\rangle}{L}
$$

Therefore (B11) is true with a probability at least

$$
1-\frac{B-2 C+C^{2}}{D^{2}}
$$

for any choice of $C$ and $D$ with $D<C$. This probability is maximal when $D \rightarrow C$ and $C \rightarrow B$ and one gets that (B11) is true with a probability at least $1 / B$. So we have shown that for $T>T_{2},(\mathrm{~B} 11)$ is true with a nonzero probability.

Now we are going to show that this probability can be made as close to 1 as needed. The idea is that by removing the first steps of the walk, we can make $B$ in (B6) as close to 1 as needed. To do this, let us first write the inequality

$$
e^{-n a / T} Y\left(L-n, \frac{n}{d}\right)<Z_{L}<(2 d)^{n} e^{n a / T} Y(L-n, n)
$$

where $Y\left(L^{\prime}, n\right)$ is the partition function of a walk of $L^{\prime}$ steps starting anywhere in a finite hypercube of linear size $2 n$ and $a$ is a bound on the energy $\varepsilon$ of each site [so the support of $\rho(\varepsilon)$ is assumed to be bounded with $|\varepsilon|<a]$. For the upper bound of $(\mathrm{B} 13), \exp (n a / T)$ is larger than the energy 
contribution of the first $n$ steps of each walk; each walk after $n$ steps belongs to the cube centered at 0 and of linear size $2 n$ and the number of walks of $n$ steps reaching each point of this hypercube is less than $(2 d)^{n}$. The lower bound can be obtained in the same way: the energy contribution of the first $n$ steps is clearly larger than $\exp (-a n / T)$, whereas the number of walks reaching each point of the cube of linear size $n / d$ and of center 0 is at least 1.

One can calculate $\left\langle(Y(L, n))^{2}\right\rangle /\langle Y(L, n)\rangle^{2}$ in the same way as was done for $\left\langle Z^{2}\right\rangle /\langle Z\rangle^{2}$ [see Eqs. (B3)-(B7)]. One finds that

$$
\lim _{L \rightarrow \infty} \frac{\left\langle(Y(L, n))^{2}\right\rangle}{\langle Y(L, n)\rangle^{2}}=B_{n}
$$

where

$$
B_{n}=1-Q_{n}+Q_{n} \frac{\left\langle e^{2 \varepsilon / T}\right\rangle /\left\langle e^{\varepsilon / T}\right\rangle^{2}}{1-A\left\langle e^{2 \varepsilon / T}\right\rangle /\left\langle e^{\varepsilon / T}\right\rangle^{2}}
$$

where $Q_{n}$ is the probability that two random walks starting at two random points in a hypercube of linear size $2 n$ will ever meet. This probability $Q_{n}$ can be calculated exactly from the theory of random walks. ${ }^{(37)}$ It is clear that in $d>2$, the further apart the starting points are, the smaller the probability is that the two random walks will ever meet. So for $n$ large enough, $Q_{n}$ can be as small as needed, and $B_{n}$ can be as close to 1 as needed.

Therefore, for $n$ large enough, using the same reasoning which leads from (B6) to (B8), one gets from (B14)

$\lim _{L \rightarrow \infty} \frac{\log Y(L, n)}{L}=\lim _{L \rightarrow \infty} \frac{\log \langle Y(L, n)\rangle}{L}$ with probability at least $\frac{1}{B_{n}}$

Then from (B13) and using the fact that

$$
\langle Y(L, n)\rangle=(2 n)^{d}\left\langle Z_{L}\right\rangle
$$

one gets that

$$
\frac{\log Z_{L}}{L}=\frac{\log \left\langle Z_{L}\right\rangle}{L}
$$

with a probability $1 / B_{n}$, where $B_{n}$ can be as close to 1 as needed. This proves (149). 


\section{ACKNOWLEDGMENTS}

J. C. acknowledges the CEN Saclay for their hospitality. We thank R. B. Griffiths, Y. Pomeau, and H. Spohn for useful discussions.

\section{REFERENCES}

1. B. Derrida and H. Spohn, J. Stat. Phys. 51:817 (1988).

2. B. K. Chakrabarti and J. Kertesz, Z. Phys. B 44:211 (1981).

3. K. Kremer, Z. Phys. B 45:149 (1981).

4. B. Derrida, J. Phys. A 15:L119 (1982).

5. A. B. Harris, $Z$. Phys. $B$ 49:347 (1983).

6. J. P. Nadal and J. Vannimenus, J. Phys. (Paris) 46:17 (1985).

7. A. K. Roy and B. K. Chakrabarti, J. Phys. A 20:215 (1987).

8. S. B. Lee and H. Nakaniski, Phys. Rev. Lett. 61:2022 (1988).

9. M. E. Cates and R. Ball, J. Phys. (Paris) 49:2009 (1988).

10. S. F. Edwards and Y. Chen, J. Phys. A $21: 2963$ (1988).

11. J. Machta and R. A. Guyer, Preprint (1988).

12. J. Machta, Phys. Rev. A 40:1720 (1989).

13. D. A. Huse and C. L. Henley, Phys. Rev. Lett. 54:2708 (1985).

14. T. Natterman, Europhys. Lett. 4:651 (1987).

15. M. Kardar, J. Appl. Phys. 61:3601 (1987).

16. L. B. Ioffe and V. M. Vinokur, J. Phys. C 20:6149 (1987).

17. T. Natterman and W. Renz, Phys. Rev. B 38:5184 (1988).

18. M. V. Feigel'man and V. M. Vinokur, Preprint (1988).

19. T. Halpin-Healy, Phys. Rev. Lett. 62:442 (1989).

20. D. Dhar, Phys. Lett. A 130:308 (1988).

21. Y. C. Zhang, Phys. Rev. Lett. 59:2125 (1987).

22. J. Rhyner and G. Blatter, to appear in Phys. Rev. B.

23. B. Derrida and R. B. Griffiths, Europhys. Lett. 8:111 (1989).

24. M. Kardar and Y. C. Zhang, Phys. Rev. Lett. 58:2087 (1987).

25. D. A. Huse, C. L. Henley, and D. S. Fisher, Phys. Rev. Lett. 55:2924 (1985).

26. M. V. Feigel'man and V. M. Vinokur, Phys. Rev. Lett. 61:1139 (1988).

27. J. Z. Imbrie and T. Spencer, J. Stat. Phys. 52:609 (1988).

28. J. P. Eckmann and C. E. Wayne, J. Stat. Phys. 50:853 (1988).

29. M. Mezard, G. Parisi, and M. A. Virasoro, Spin Glass Theory and Beyond (World Scientific, Singapore, 1987).

30. G. Parisi, Phys. Rev. Lett. 50:1946 (1983).

31. M. Mezard, G. Parisi, N. Sourlas, G. Toulouse, and M. Virasoro, J. Phys. (Paris) 45:843 (1984).

32. B. Derrida, Phys. Rev. B 24:2613 (1981).

33. G. Toulouse and J. Vannimenus, Phys. Rep. 67:47 (1980).

34. A. N. Berker and S. Ostlund, J. Phys. C 12:4961 (1981).

35. R. B. Griffiths and M. Kaufman, Phys. Rev. B 26:5022 (1982).

36. M. Kardar, Phys. Rev. Lett. 55:2923 (1985).

37. E. W. Montroll and B. J. West, in Fluctuation Phenomena, E. W. Montroll and J. L. Lebowitz, eds. (North-Holland, Amsterdam, 1979), p. 61. 